\title{
Design and Configuration of a Tridyne Propulsion System for CubeSat Applications
}

\author{
Peter J. Sciuto III
}

\author{
A thesis \\ submitted in partial fulfillment of the \\ requirements for the degree of
}

Master of Science

University of Washington

2021

Committee:

James Hermanson, Chair

Carl Knowlen

Program Authorized to Offer Degree:

Aeronautics \& Astronautics 
(C) Copyright 2021

Peter J. Sciuto III 


\title{
University of Washington
}

\author{
Abstract \\ Design and Configuration of a Tridyne Propulsion System for CubeSat Applications \\ Peter J Sciuto III \\ Chair of the Supervisory Committee: \\ James Hermanson \\ Department of Aeronautics \& Astronautics
}

A warm-gas thruster using a catalyzed tridyne propellant (88\% N2, 8\% H2 and 4\% 02 by volume) is developed at the University of Washington in collaboration with Aerojet Rocketdyne for the purposes of advancing small-scale satellite propulsion. A detailed design of a $1.5 \mathrm{U}(10 \mathrm{~cm} \times 10 \mathrm{~cm} \times 15 \mathrm{~cm})$ CubeSat propulsion unit has been developed in a configuration suitable for space applications. The design incorporates accumulator volumes to allow for pressure regulation as well as pulse-modulating the thrust chamber pressure in a "bang-bang" fashion, has all welded joints for space application, and has all material compatibility issues addressed. Though the primary focus of the effort is the development of a thruster configuration for integration into a CubeSat form factor, the work also includes testing of the thruster configuration, including successful demonstration of the bang-bang pressure control system. 


\section{Table of Contents}

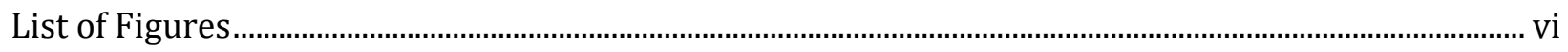

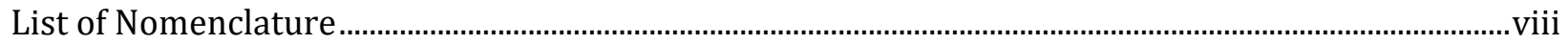

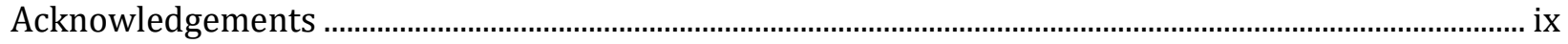

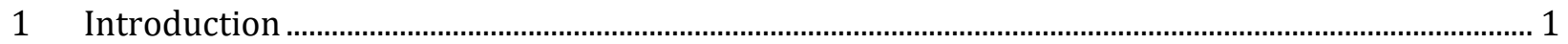

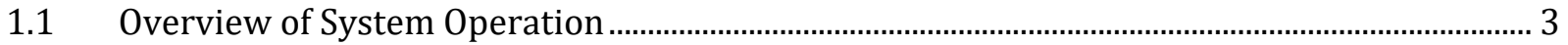

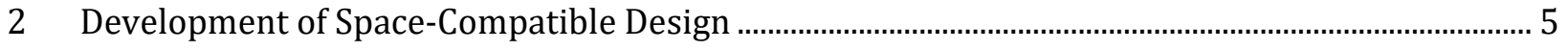

$2.1 \quad$ Overview of Design and Constraints .......................................................................................... 5

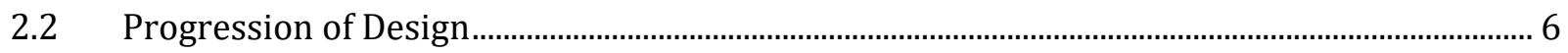

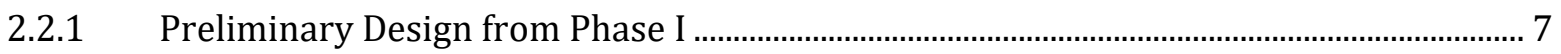

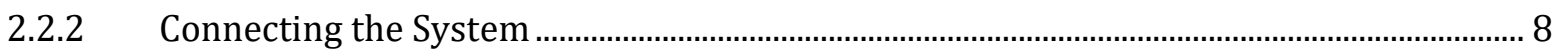

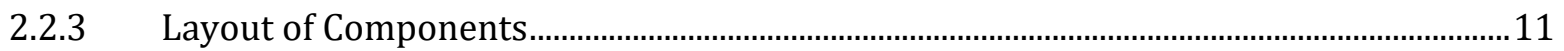

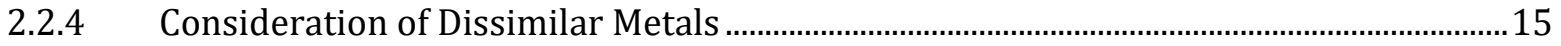

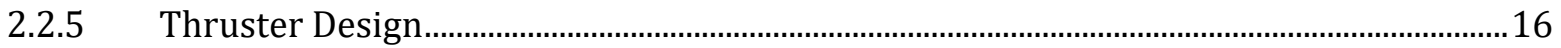

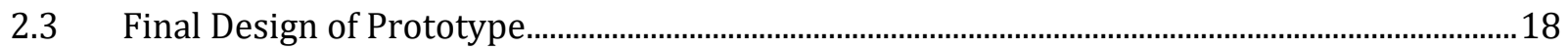

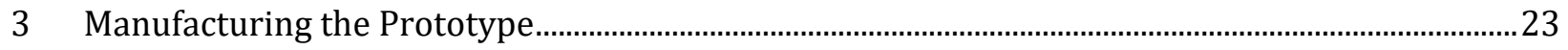

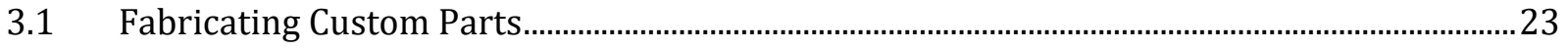

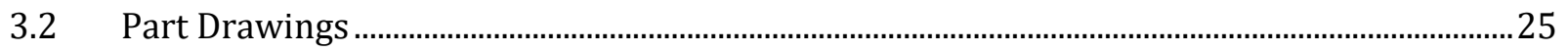

3.3 Assembly Tooling and System Assembly ……........................................................................... 26

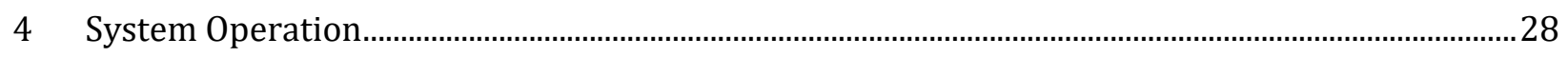

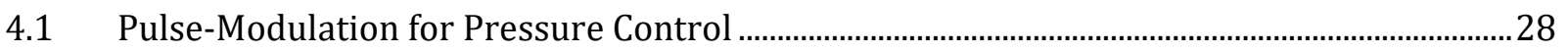

4.2 Calculated System Pressures and Valve Timing ............................................................................. 29

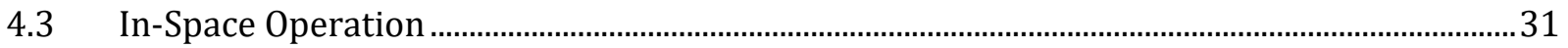

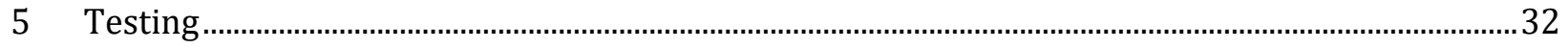

$5.1 \quad$ Benchtop Testing of Pulse-Modulated Operation ............................................................................. 32

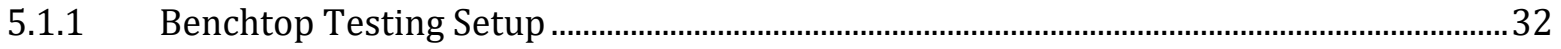




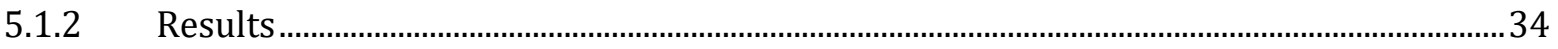

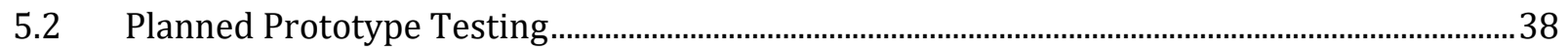

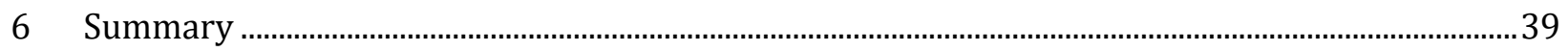

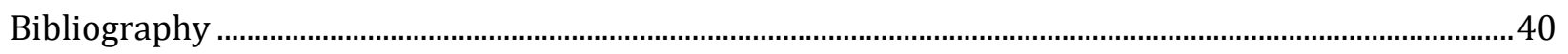

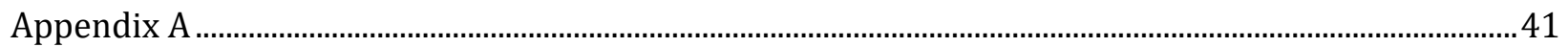

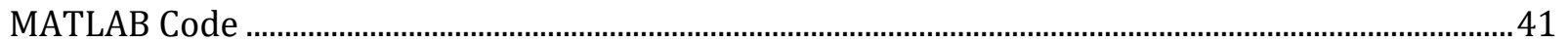




\section{List of Figures}

Figure 1 - CubeSat launches in recent years. Reproduced from [2] -

Figure 2 - Tridyne gas reacting as it flows over a platinum-iridium catalyst bed [3]-------------------- 2

Figure 3 - Final PID of the prototype system. ---:- 3

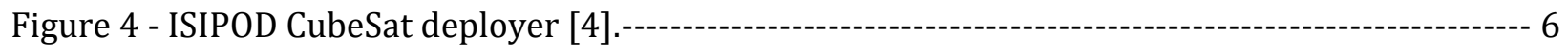

Figure 5 - $1.5 \mathrm{U}$ footprint plus tuna-can volume (dimensions in $\mathrm{cm}$ ). ----------------------------------- 6

Figure 6 - Initial representative system design (CAD by Brian Henderson) [3] ------------------------- 7

Figure 7 - Final design of the tank mounting tabs and component plate. ------------------------------- 8

Figure 8 - Tab style mounting points on the tank (A, B) and potential component plate designs (C, D).

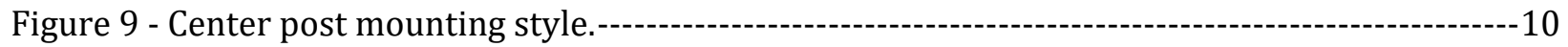

Figure 10 - U-bracket mounting (left) and potential component plate design (right). ----------------10

Figure 11 - Vertical valve placement within the tuna-can solved many issues. -------------------------11

Figure 12 - First reconfiguration of components. ---

Figure 13 - Component layout with first inclusion of an increased plenum volume, mounting

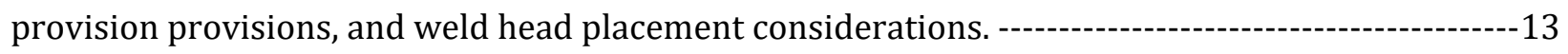

Figure 14 - Larger accumulators, new mounting, and fully weldable connections. --------------------14

Figure 15 - Titanium to CRES reducing union, with titanium on the left and CRES on the right. -----15

Figure 16 - Cross section of the transitional reducing union, showing the internal FOD filter. -------16

Figure 17 - Cross section of Phase I thruster design [3].----

Figure 18 - Final thruster assembly without heat shield (left), and cross section of thruster assembly

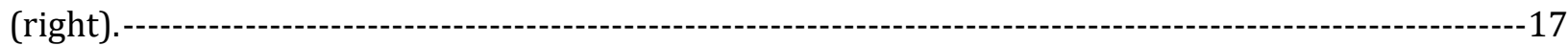

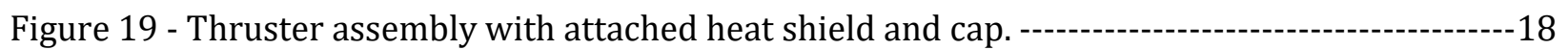

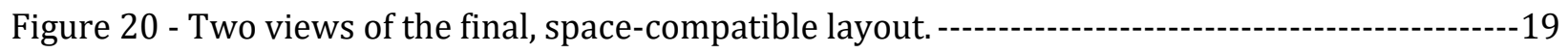

Figure 21 - Final design with labeled components. ---

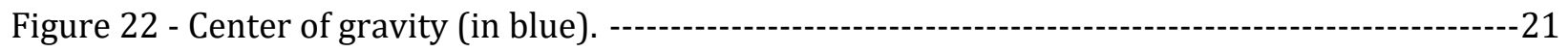

Figure 23 - 3-D renders at different angles representing the final, space-compatible system design. $-22$

Figure 24 - Fusion360 setup showing the stock metal and tooling plate within (left), and a single step

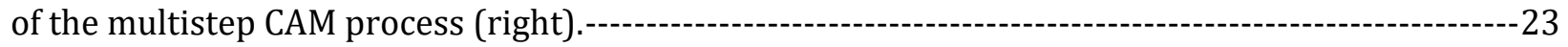

Figure 25 - Shot of the tooling plate being machined on a CNC mill. --------------------------------24

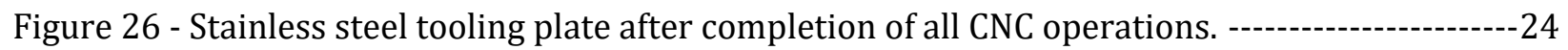

Figure 27 - 2-D drawing of the thruster standoff. --- 
Figure 28 - Thruster assembly and valves bolted to the tooling plate/stand for welding.-------------26 Figure 29 - The order of welding (left), and ensuring the weld head has clearance for all welds (right).

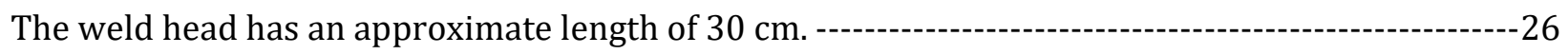

Figure 30 - After bolting the plate to the tank, the final weld is performed. ----------------------------27

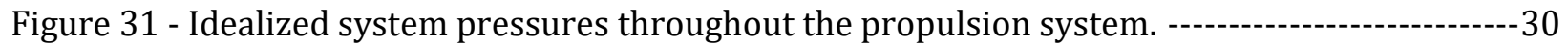

Figure 32 - Bench top setup with modular accumulator volumes on a 4' optical breadboard. -------33

Figure 33 - Circuit diagram for single valve control with an Arduino Uno [6]. -----------------------33

Figure 34 - Breadboard wiring connected to an Arduino Uno for dual valve control. -----------------34

Figure 35 - Filtered pressure response with a 500 psi source, the downstream valve open, and the upstream valve pulsing with a square-wave input. Recall that accumulator 1 is the upstream plenum volume between the two valves, and accumulator 2 is the plenum volume just upstream of the

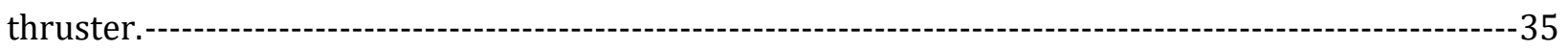

Figure 36 - Average system pressures as the square wave frequency is varied for the upstream valve,

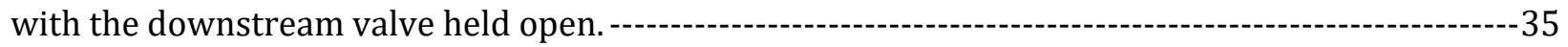
Figure 37 - Changing the duty cycle of the PWM signal allows downstream pressures to be controlled. The valve's open time was held constant at $100 \mathrm{~ms}$ for each run. --Figure 38 - System response to downstream valve duty cycle changes for PWM signal. --------------36

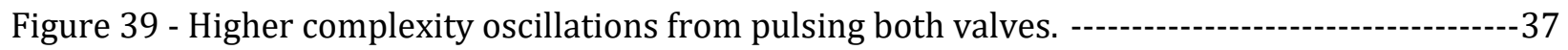




\section{List of Nomenclature}

$U$

$N$

$I_{s p}$

$I_{\text {tot }}$

$\Delta V$

P

$T$

$R$

$\alpha$

$\gamma$

TRL

PID

CAD

CG

CRES

FOD

FoS

CAM

CNC

MMU

DAQ

MOSFET

PWM
Standard CubeSat unit size

Newton, unit of force

Specific impulse

Total impulse

Change in velocity

Pressure

Temperature

Gas constant

Duty cycle

Ratio of specific heats

Technology Readiness Level

Plumbing and Instrumentation Diagram

Computer-Aided Design

Center of Gravity

Corrosion-Resistant Steel

Foreign Object Debris

Factor of Safety

Computer-Aided Manufacturing

Computer Numerical Control

Manned Maneuvering Unit

Data Acquisition

Metal-Oxide-Semiconductor Field-Effect

Transistor

Pulse-Width Modulation $\left[10 \times 10 \times 10 \mathrm{~cm}^{3}\right]$

$\left[\mathrm{kg} \mathrm{m} / \mathrm{s}^{2}\right]$

$[s]$

$[N s]$

$[\mathrm{m} / \mathrm{s}]$

[psi]

$[K]$

$[\mathrm{Jg} K]$ 


\section{Acknowledgements}

Thank you to my fantastic advisors, Dr. James Hermanson and Dr. Carl Knowlen for the opportunity to work on this fascinating project, and for their time and guidance. I would also like to thank Brian Henderson for all the prior work he did on this project, providing a solid foundation upon which to design this system. Lastly, I would like to thank my fiancé, Brittany, for all of her love and support over the last year.

This work is supported by the Washington State Joint Center for Aerospace Technology Innovation (JCATI), and our industry partner Aerojet Rocketdyne of Redmond, WA. Special thanks to Messrs. Christopher Sebastian and Matthew Jakubek, Dr. Dieter Zube and all the other Aerojet Rocketdyne folks who provided their time and engineering expertise, and taught me so much along the way. 


\section{Introduction}

CubeSats are small satellites that have seen an exponential growth in use in recent years, and are used for educational, commercial, and governmental purposes. Some common uses are scientific investigations, new technology demonstrations, and increasingly, for communications as well. The size designation of a CubeSat is called a " $U$ " which is a $10 \times 10 \times 10 \mathrm{~cm}^{3}$ cube. CubeSats come in various multiples of U's, like $1.5 \mathrm{U}, 2 \mathrm{U}, 6 \mathrm{U}$ all the way up to $24 \mathrm{U}$. This configurability, along with their low mass and size, make CubeSats a good alternative to traditional satellites. Additionally, the cost of launching a CubeSat is low by comparison, with the cost of launching a CubeSat estimated at about $\$ 30,000$ per kilogram [1], making these satellites affordable for educational research and smaller companies.

Launches of CubeSats began in the early 2000's, and have been increasing yearly since, likely due to the customizable form factor and low cost of launch [2]. The rapid growth of the CubeSat market from 2006 to 2018, along with the purpose of the mission, is shown in Fig. 1. This increase in use of CubeSats has created a new market for CubeSat propulsion systems. Including a propulsion system onboard allows CubeSat maneuvering, including attitude control for maintaining orientation or constellation positioning, station keeping to prevent orbital decay, and potentially deorbiting at

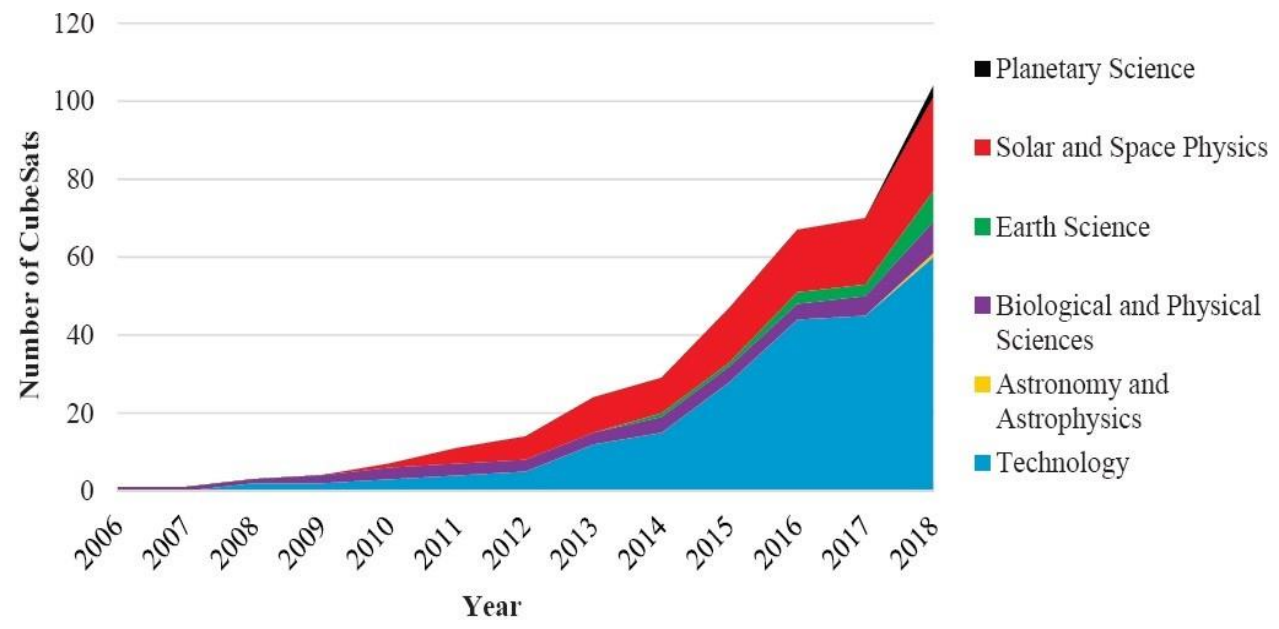

Figure 1 - CubeSat launches in recent years. Reproduced from [2]. 
the end of a mission. Recently, there has been interest in utilizing CubeSats for interplanetary missions.

Typical propulsion systems for CubeSats provide a wide range of both thrust and specific impulse $\left(I_{s p}\right)$, depending on the whether the system is cold-gas, chemical, or electric propulsion. Cold gas systems are simple, but provide low $I_{s p}$ and thrust. Chemical systems provide higher $I_{s p}$ and thrust, but are more complex. Electric propulsion systems have a very high $I_{s p}$ but low thrust and high system complexity, making these systems only suitable in certain applications. Prior work done during Phase I of this effort demonstrated the need for a new propulsion system that provided the simplicity of a cold gas system but the performance equal to or greater than a more complex liquid chemical system [3].

The propulsion system designed herein meets that need, and is a joint effort between the University of Washington (UW) and Aerojet Rocketdyne (AR), with funding provided by the Joint Center for Aerospace Innovation Technology (JCATI). The system is a "warm-gas" thruster that makes use of tridyne gas ( $88 \% \mathrm{~N} 2,8 \% \mathrm{H} 2$ and 4\% 02 - diluent, fuel and oxidizer, respectively) as a propellant. This propellant is environmentally friendly, safe to handle, and inert at room temperatures. The primary advantage of the propellant is that it can be self-heated by reacting with a platinum-iridium catalyst, prior to expanding through a nozzle. A representative illustration of the tridyne gas flowing over the catalyst bed in a thrust chamber, heating as it flows downstream, is shown in Fig. 2. The propulsion system itself is simple, low-cost, provides quasi-steady operation, is

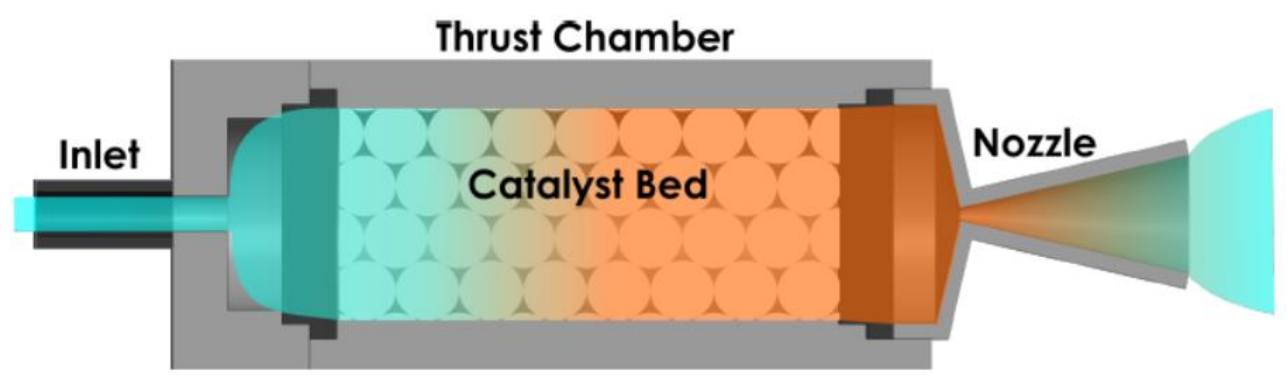

Figure 2 - Tridyne gas reacting as it flows over a platinum-iridium catalyst bed [3]. 
fully restartability, with no need for external heating. The system design presented is intended to provide up to $1 \mathrm{~N}$ of thrust with an $I_{s p}$ of $140 \mathrm{~s}$ and $I_{\text {tot }}$ of about $550 \mathrm{~N}-\mathrm{s}$, with a propellant storage pressure of 10,000 psi and chamber pressure of 200 psi [3].

The current Technology Readiness Level (TRL) of this tridyne propulsion system is between TRL 5 and 6, in that component and breadboard validation has been successfully completed, and a prototype system has been manufactured for assembly and testing. The primary objectives of this effort have been to design, configure and build a CubeSat compatible tridyne propulsion system prototype, that fits within a $1.5 \mathrm{U}$ volume.

\subsection{Overview of System Operation}

The system simplicity is evident when viewing the plumbing and instrumentation diagram (PID) shown in Fig. 3. The primary components are a custom, 3-D printed titanium tank (designed and printed by AR), two high-speed solenoid valves for rapid, pulsed control, a pressure transducer, two accumulators to provide increased plenum volumes, and a catalyst-packed thrust chamber attached to an exhaust nozzle. Due to size and reliability concerns, a pressure regulator is not used and instead, the two solenoid valves are operated in a bang-bang configuration to sequentially step

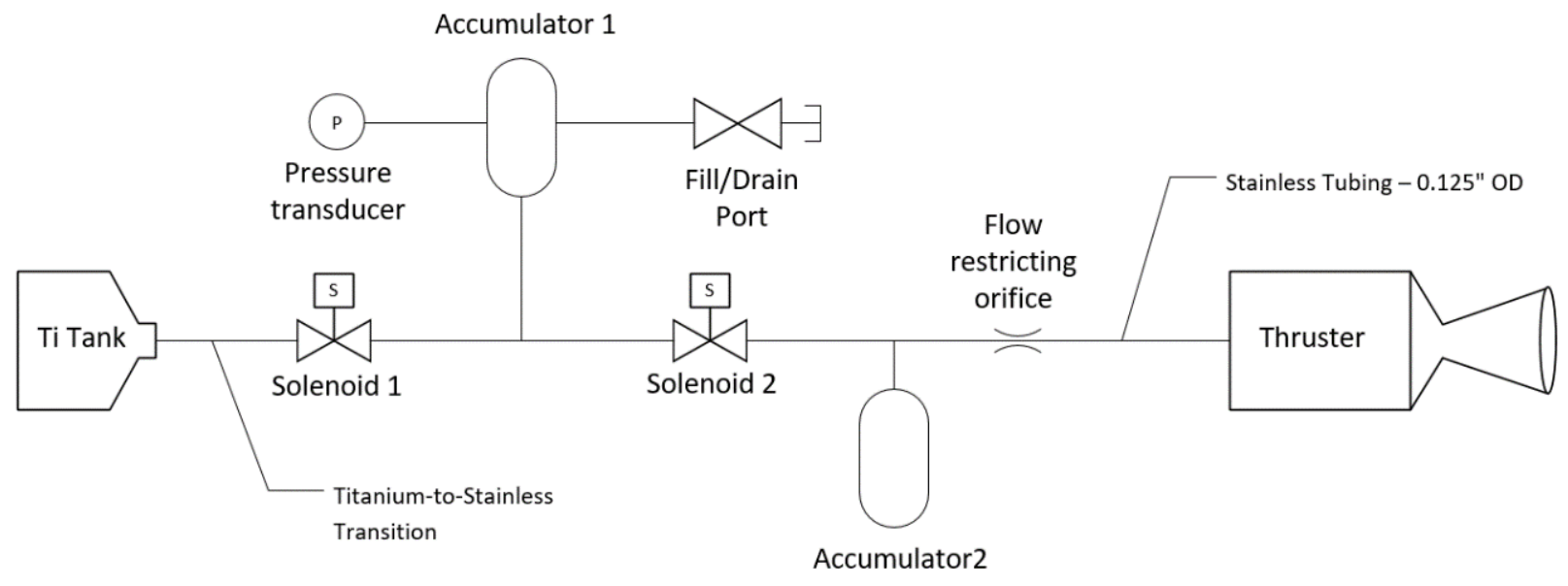

Figure 3 - Final PID of the prototype system. 
the propellant pressure down from the storage pressure of 10,000 psi to a quasi-steady chamber pressure of approximately 200 psi. This gives the additional benefits of providing precise impulse control, and allows the system to be restarted as needed. Further, the valves can be dynamically controlled such that the chamber pressure will remain roughly constant as the stored propellant pressure decreases, until the storage pressure is equal to the chamber pressure. 


\section{Development of Space-Compatible Design}

\subsection{Overview of Design and Constraints}

Several major constraints drove the design for the prototype, namely the need to store tridyne gas at a pressure of up to 10,000 psi, the small amount of available space for components within a $1.5 \mathrm{U}$ footprint, and the need to minimize the number of components used to decrease potential failure points.

Work done in Phase I of this effort showed that with the chosen mix of tridyne propellant, a supply of 10,000 psi would make the system competitive with other state-of-the-art CubeSat propulsion systems [3]. Both custom and off-the-shelf components were designed or selected with these pressure requirements in mind. The chosen operating pressure also informed the material choice for the Aerojet Rocketdyne designed, custom pressure vessel. This tank needed to be strong, lightweight and 3-D printable, so titanium was chosen. The remaining components and plenums are made of stainless steel or Inconel, so a transition from titanium to stainless steel was necessary in the design. Additionally, in order to operate the valves in a bang-bang configuration at high operating pressures, additional plenum volumes were needed to decrease the required speed of the valves.

In order to provide the greatest total impulse, the propellant storage tank takes up most of the available $1.5 \mathrm{U}$ volume, leaving relatively little space for the remaining components. To optimize space utilization, the typical "tuna-can" volume found in standard deployment systems was used. Fig. 4 shows a typical CubeSat deployment system and Fig. 5 shows a $1.5 \mathrm{U}$ footprint with the addition of the tuna-can volume, where the tuna-can would sit within the deployment unit. 


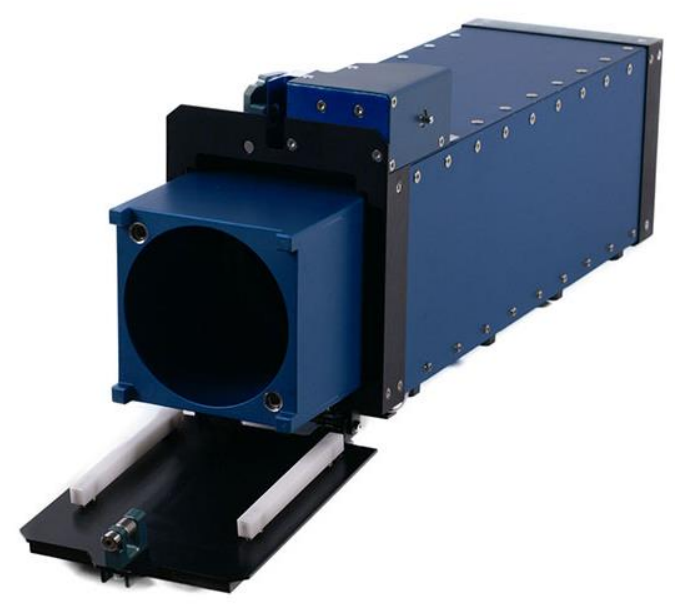

Figure 4 - ISIPOD CubeSat deployer [4].
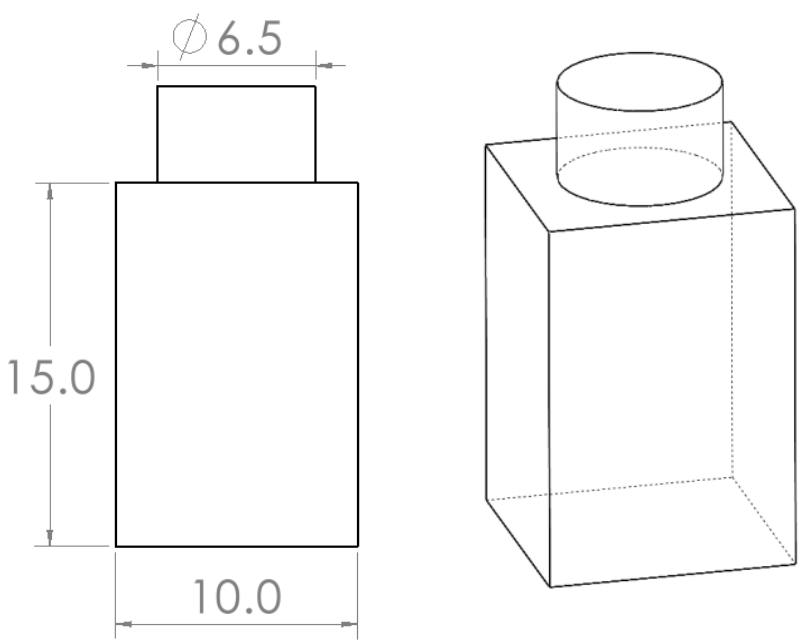

Figure 5 - 1.5 U footprint plus tuna-can volume (dimensions in $\mathrm{cm}$ ).

Fully-welded joints are employed instead of fittings to decrease the number of total parts, increase simplicity, and decrease potential points of failure. This added a requirement that all 1/8" tube joints have 0.25 " of unobstructed tubing on either side, allowing for the positioning of an orbital weld head assembly. Fitting and properly spacing all the components in a fashion that minimized their footprint was the main design challenge to overcome. After multiple iterations, a suitable layout was found that minimized the system footprint and plenum complexity, maximized accumulator volumes for ideal bang-bang operation, and allowed for system assembly and welding. Iterations of the design are presented and discussed in Section 2.2.

\subsection{Progression of Design}

Settling on a final design was a months-long, iterative effort that required substantial collaboration between UW and AR. Many different concepts were considered for mounting, connecting, and laying out components, all of which happened concurrently, though the following sub-sections will discuss individual aspects of the design sequentially. 

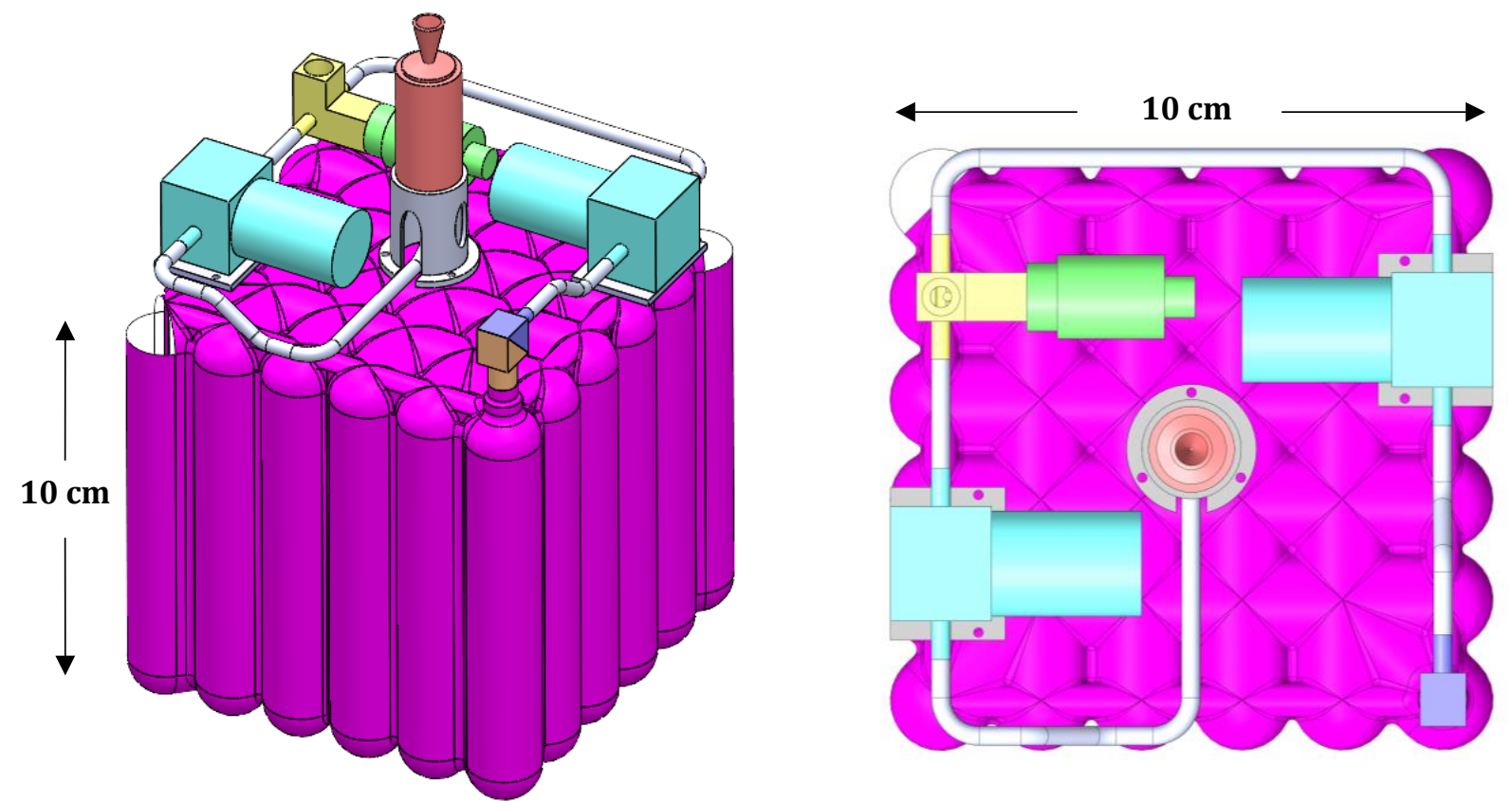

Figure 6 - Initial representative system design (CAD by Brian Henderson) [3].

\subsubsection{Preliminary Design from Phase I}

The primary focus of Phase I was to determine the viability of a tridyne propulsion system as a competitive propulsion option for CubeSats, but some initial representative computer-aided design (CAD) was performed to get a sense of how the system might fit together. This initial design provided a good starting point for Phase II, but had not yet addressed some of the major design constraints. Fig. 6 shows this initial design with the AR designed pressure vessel (pink), solenoid valves (cyan), fill/drain port (yellow), pressure transducer (green) and thruster (orange). At this point, the need for increased plenum volumes was not known, so no accumulators were included. Additionally, mounting the components to the tank had not been considered, and the tube bends are overly complex and would be difficult to fabricate. 


\subsubsection{Connecting the System}

One of the first items considered was how to connect the individual propulsion system components to the 3-D printed tank. Three initial mounting concepts considered, including tab-style mounts, center post mount, and U-bracket style mounts. All three could be directly incorporated into the pressure vessel design, and were meant to be part of the 3-D print.

The tab-style mounts were ultimately selected for the final design shown in Fig. 7. The placement of both the tabs and components were considered, ensuring that the full edge-to-edge space could be used for components. A trade-off of this style is the higher complexity plate which requires machining, but the height can be set and by making the plate out of stainless steel, holes can

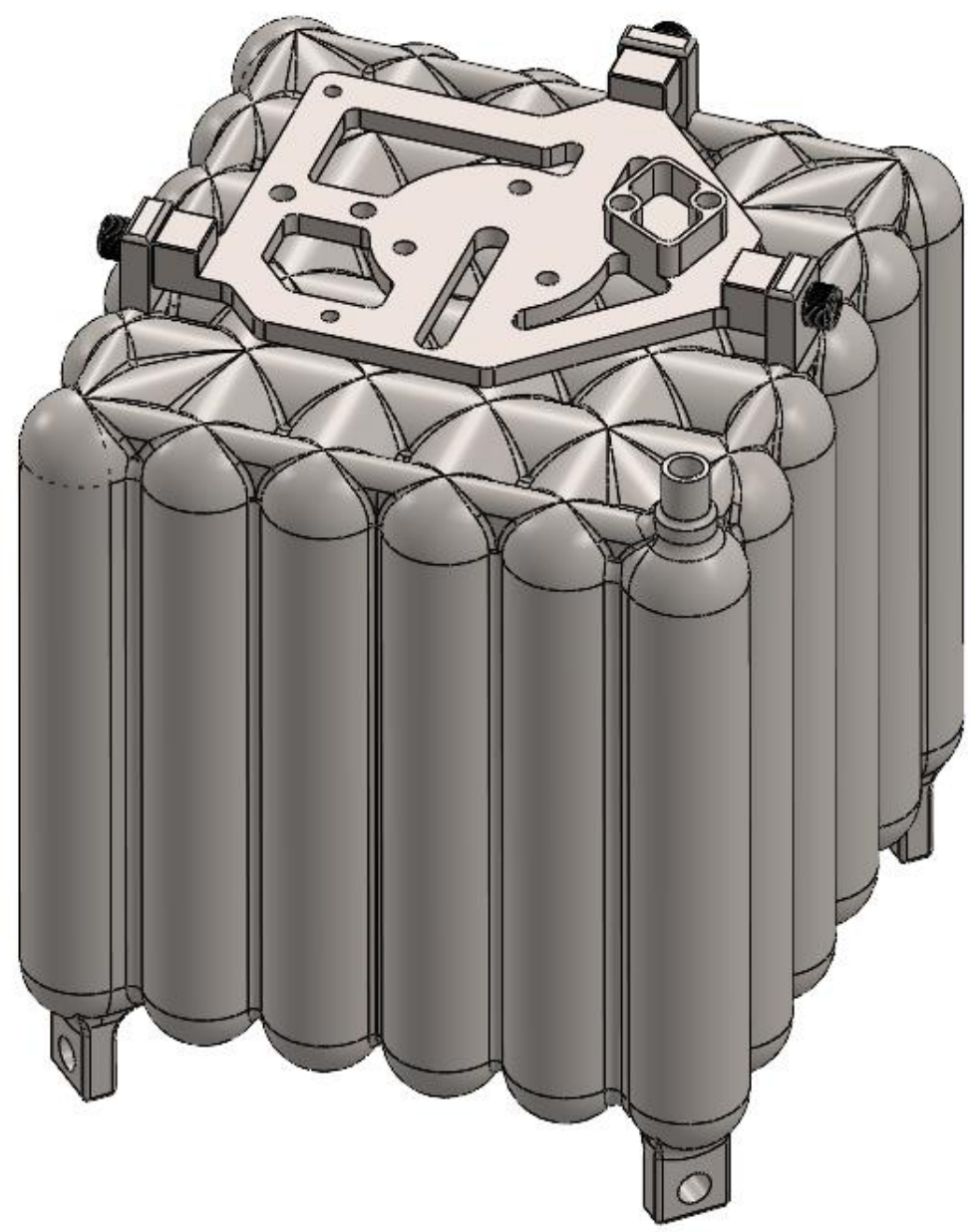

Figure 7 - Final design of the tank mounting tabs and component plate. 
be tapped which eliminates the need for nuts when bolting components to the plate. Note that tabs were added to the bottom of the tank as well, to show that connecting the propulsion system to a customer's CubeSat has been considered. The connection style and placement used for the bottom connection points can be changed for individual customers based on their needs.

The initial tab-style extrusion design is shown in Fig. 8 along with potential component mounting plate designs, and tabs would be placed either in the corners or along the edges. This style is simple, structurally supported by the tank, and is easy to 3-D print. The main disadvantage of this design was that without consideration of component layout, precious edge space can be wasted. The bent corners make the component plate more complex, either requiring multiple sheet metal bends or machining of the entire plate. Additionally, the plate leaves either too much or too little clearance between the plate and tank, depending on its orientation.
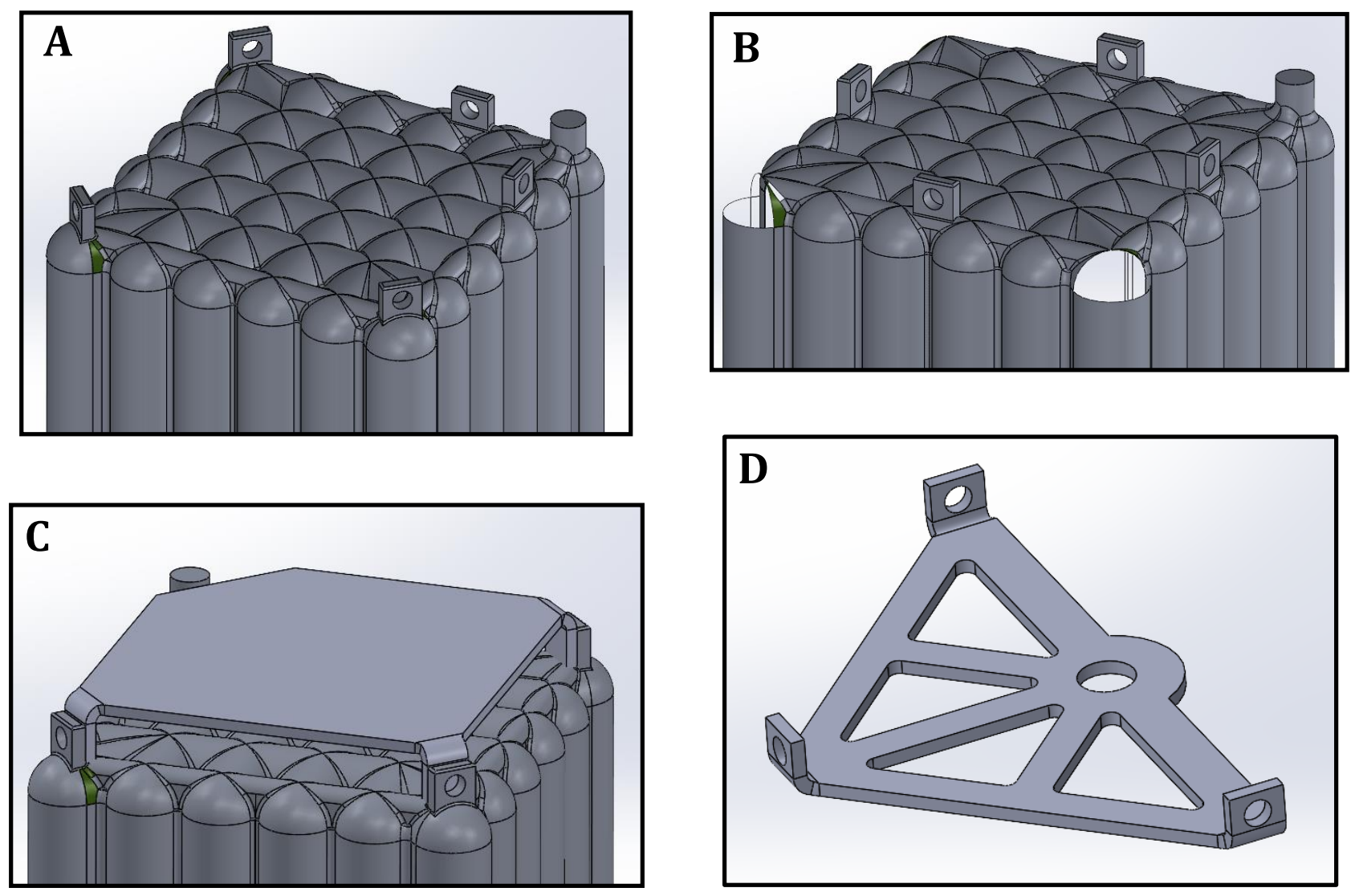

Figure 8 - Tab style mounting points on the tank $(A, B)$ and potential component plate designs $(C, D)$. 


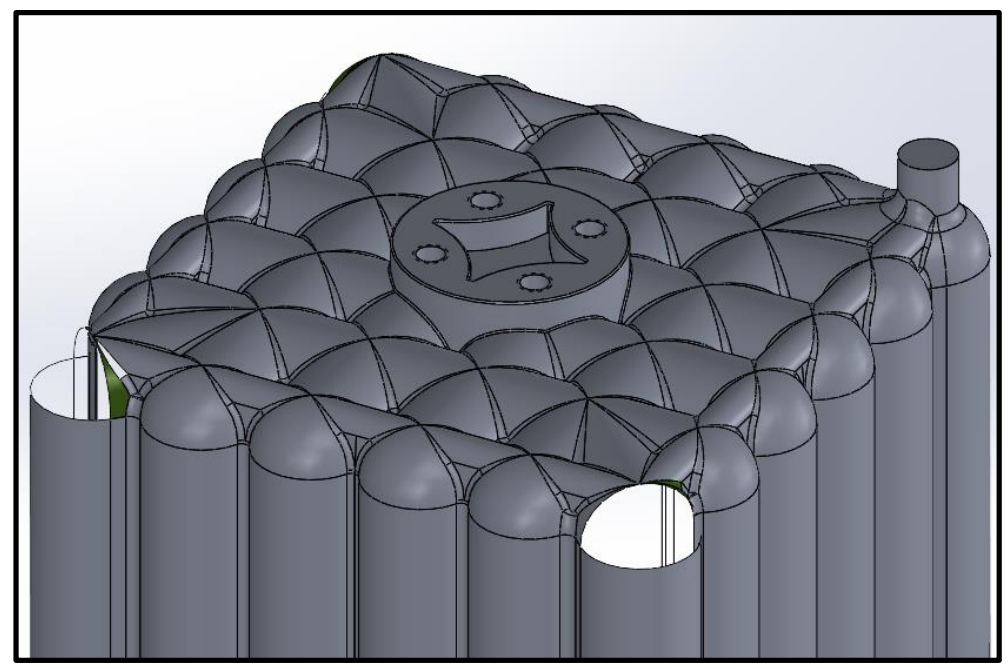

Figure 9 - Center post mounting style.

Another candidate design is the center post style mount is shown in Fig. 9. This would be easy to 3-D print, directly transfers thrust into the system body, and allows for full edge-to-edge component placement. Some downsides to this style include increased plate complexity requiring machining, poor structural support of the mounting post, and the design would require drilling into and tapping the pressure vessel.

The last mounting concept that was explored was the U-bracket style mounts shown in Fig. 10. The higher complexity plate required for this concept would allow the clearance between the plate and tank to be controlled, the mounting brackets were out of the way of the components, and
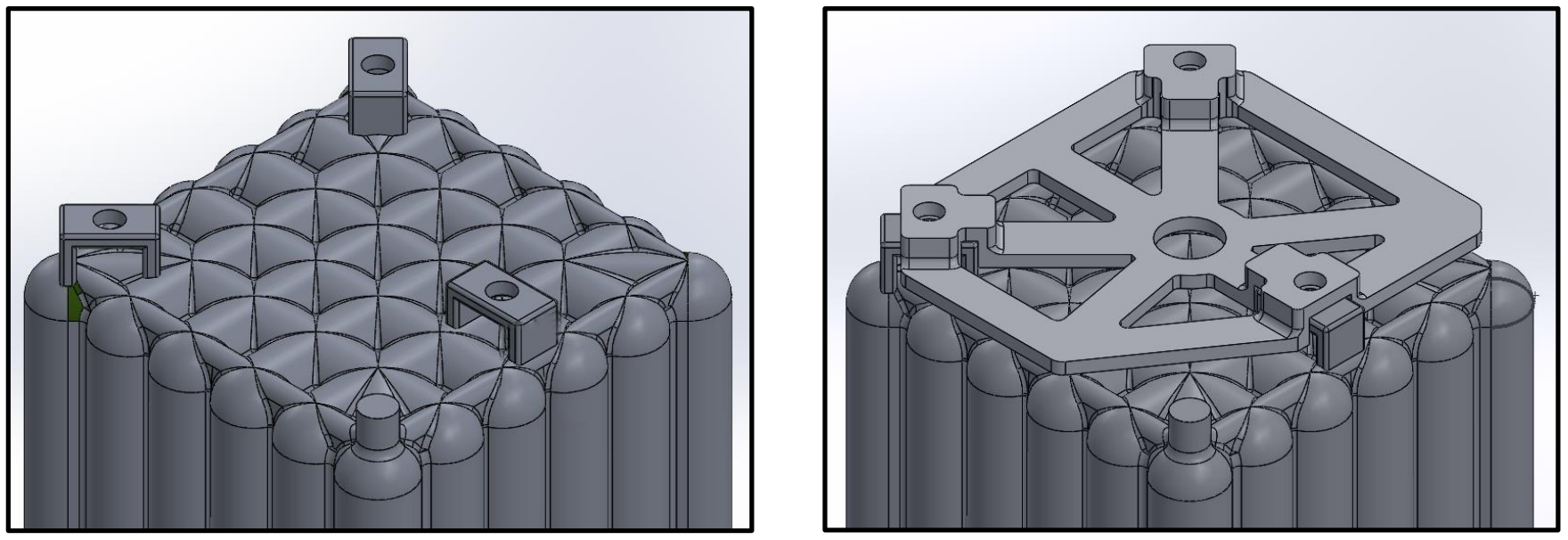

Figure 10 - U-bracket mounting (left) and potential component plate design (right). 
they are structurally supported by the tank. This concept, however, does require a complex plate, is difficult to 3-D print, and would make assembly a challenge.

\subsubsection{Layout of Components}

After establishing the base plate/propellant tank configuration, the most time-consuming portion of the design was finding the optimal placement of all the components and plenums. Discovering the need for increased plenum volumes part-way through the project further increased the difficulty of fitting everything within the $1.5 \mathrm{U}$ footprint. Layouts were created then discussed as a team, leading to quick iterations until a final design was selected. The final layout design is presented, followed by discussion of the layout progression.

The final layout presented in Fig. 11 significantly improved upon prior designs, enabled by rotating the valves and placing them vertically within the tuna-can volume, and incorporating a fill/drain port and pressure transducer into the upstream accumulator instead of as standalone components. These changes decreased the length and complexity of the tubing, decreased the
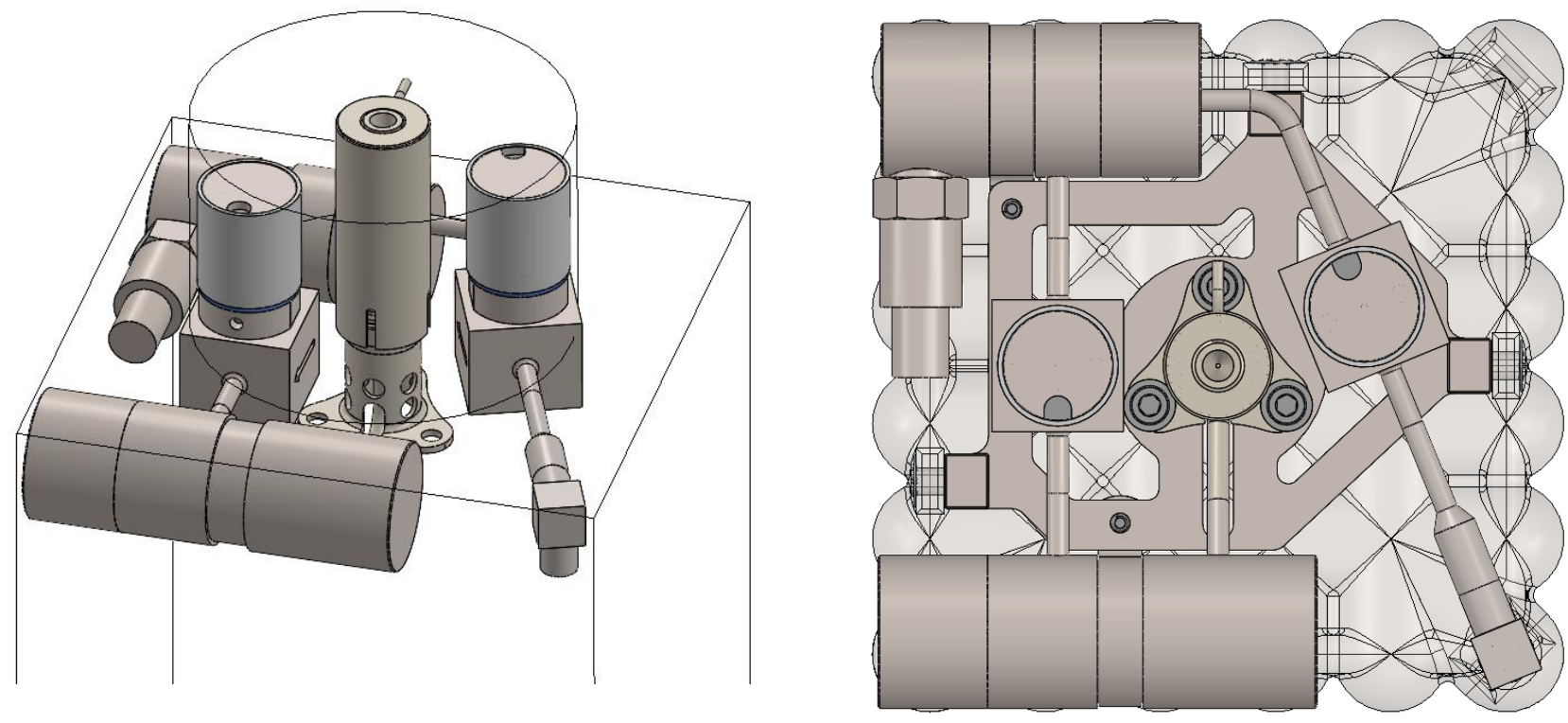

Figure 11 - Vertical valve placement within the tuna-can solved many issues. 

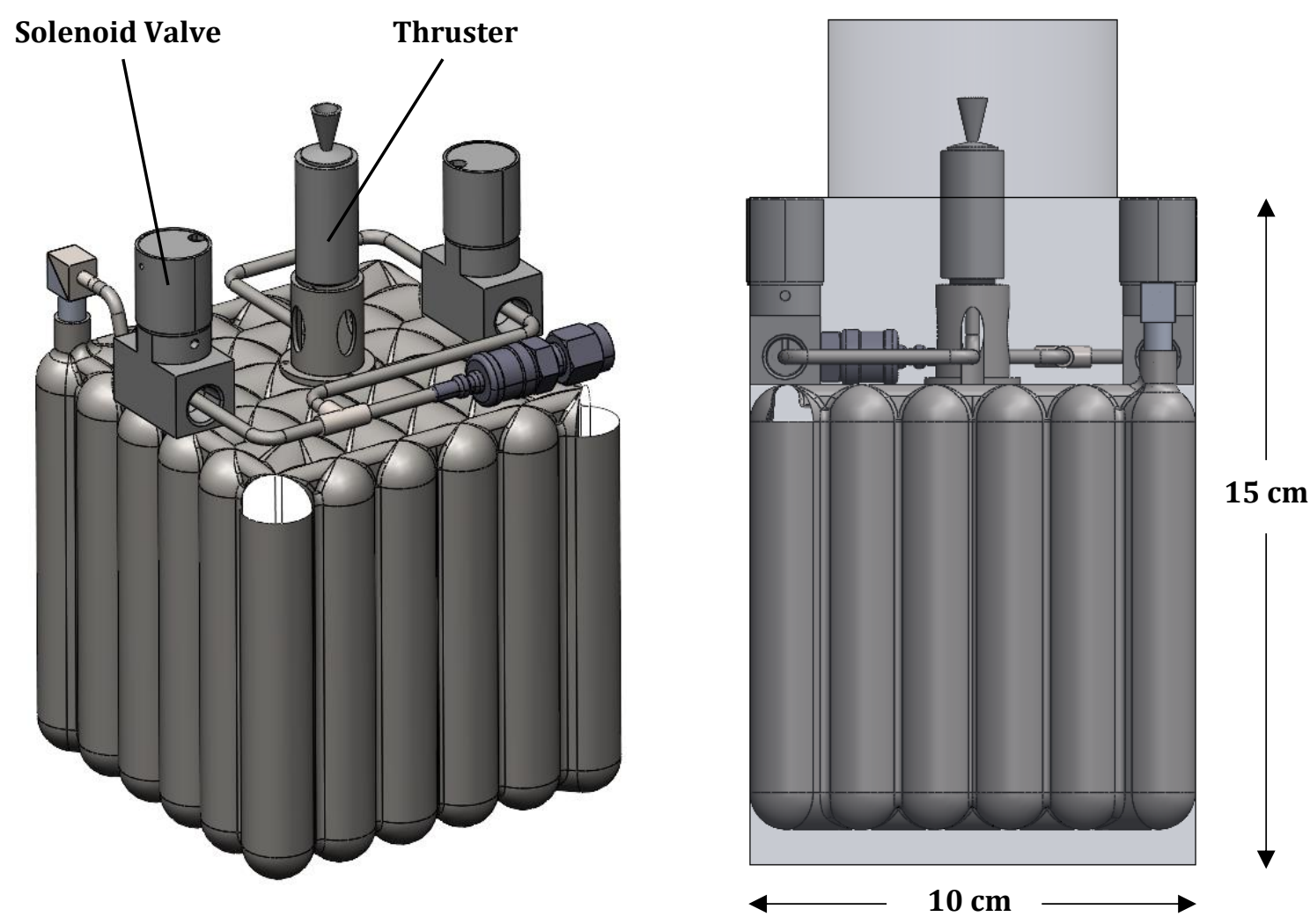

Figure 12 - First reconfiguration of components.

number of required welds, allowed for a significant increase in accumulator volumes, and still left open space for mounting to the tank. Additionally, there is room both on the component plate and in the upper corner to add optional weight for adjusting the center of gravity (CG), if needed.

The first reconfiguration done during Phase II is shown in Fig. 12 and mainly served the purpose of increasing familiarity with the project and components. This design did not yet include provisions for mounting components, and is missing some primary components such as a pressure transducer and accumulators. A quick-connect fitting was included in the design, intended to easily fill the tank, but this inclusion was ultimately deemed unnecessary and was removed in the next iteration. Additionally, the vertically-placed solenoid valves decreased space that could be otherwise used to increase the pressure vessel volume, and the tuna-can volume was underutilized. The tubing bends are complex and spacing for welding components had not been considered. Input during an all-team meeting quickly led to the next iteration. 
The second major iteration shown in Fig. 13 had significant improvements over the previous design. Basic flow calculations (discussed in Section 4.2), showed the need for increased plenum volumes in order to control pressures and operate the system in a bang-bang configuration, so a first attempt at increasing those volumes was included. Corner tab mounts and a component plate were included in the design, though it was found that there wasn't room for hardware to bolt the plate to the tank, and the corner placement did not allow components to be placed edge-to-edge. The pressure transducer and fill/drain port were added back in, though clearance was a concern. Tube bends were simplified over the previous design, but connecting components to each other had not been fully addressed. Rotating the valves sideways also decreased the volume required for components and plenums, again giving the option to increase the tank volume.
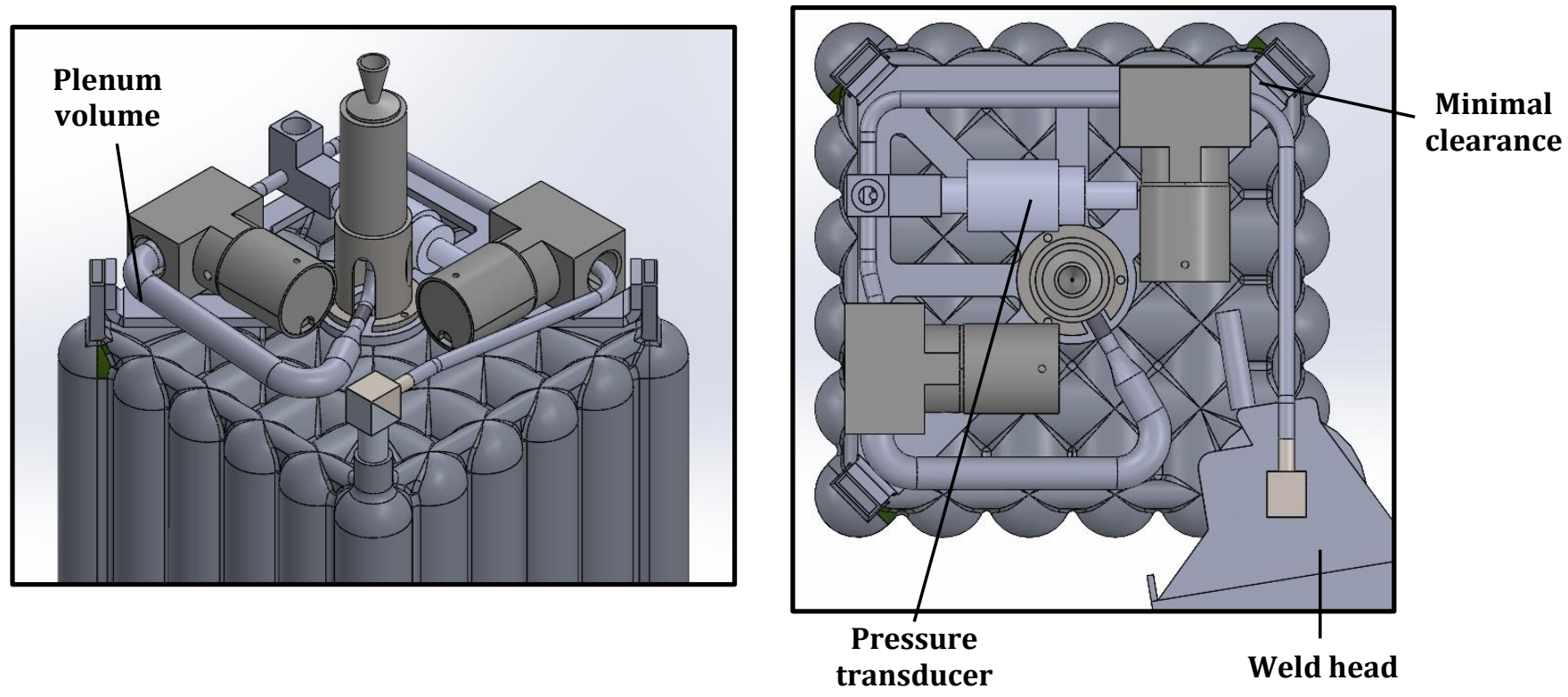

Figure 13 - Component layout with first inclusion of an increased plenum volume, mounting provision provisions, and weld head placement considerations. 


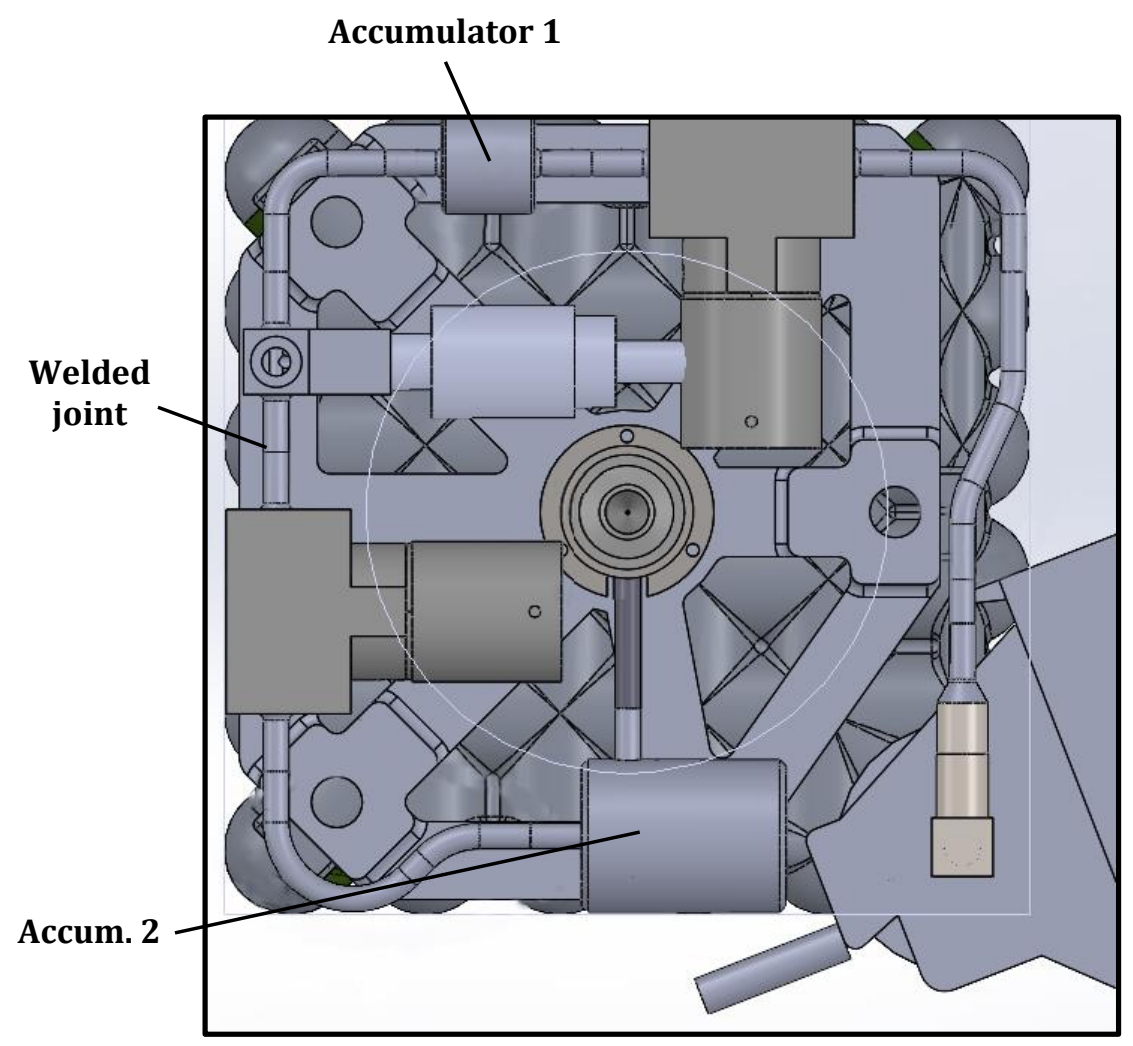

Figure 14 - Larger accumulators, new mounting, and fully weldable connections.

In the third major iteration (see Fig. 14), plenum volumes were further increased by adding two accumulators to the design, mounting was switched from tabs to U-brackets, and fully welded connections between components was considered. While the plenum volumes were increased, the size of each accumulator was limited by available space in the layout, and the volumes were smaller than the calculation indicated they needed to be. The U-bracket mounts allowed for full edge-to-edge $\left(10 \times 10 \mathrm{~cm}^{2}\right)$ utilization but ultimately were removed in the next iteration due to the 3-D printing difficulty caused by the overhangs. All the components could now be welded together, as there was now 0.25 " of space on either side of each weld, as required by the weld head, though this did increase the complexity of the tube bends. 


\subsubsection{Consideration of Dissimilar Metals}

With a titanium tank and stainless steel components, consideration had to be taken for joining these dissimilar materials while maintaining the pressure ratings of each connected component. A company, Interface Welding, was identified that specializes in joining dissimilar metals, and several cylindrical titanium to CRES (corrosion-resistant steel) blanks were purchased. The final transition piece (shown in Fig. 15) is a $1 / 4$ " to $1 / 8$ " reducing union, that transitions from titanium to CRES at the thickest part of the body. Additionally, internal features were added to allow for the inclusion of a filter to prevent FOD (foreign object debris) from flowing through the system. A cross section of the reducing union with filter installed is shown in Fig. 16. Prior to settling on the final design, attempts were made at incorporating the titanium to CRES joint into the $90^{\circ}$ elbow that connects to the tank, but the design quickly became overly complex with the available blank transition pieces. Ultimately, after the valves were rotated and placed within the tuna-can, there was room in the layout to incorporate the separate reducing union.

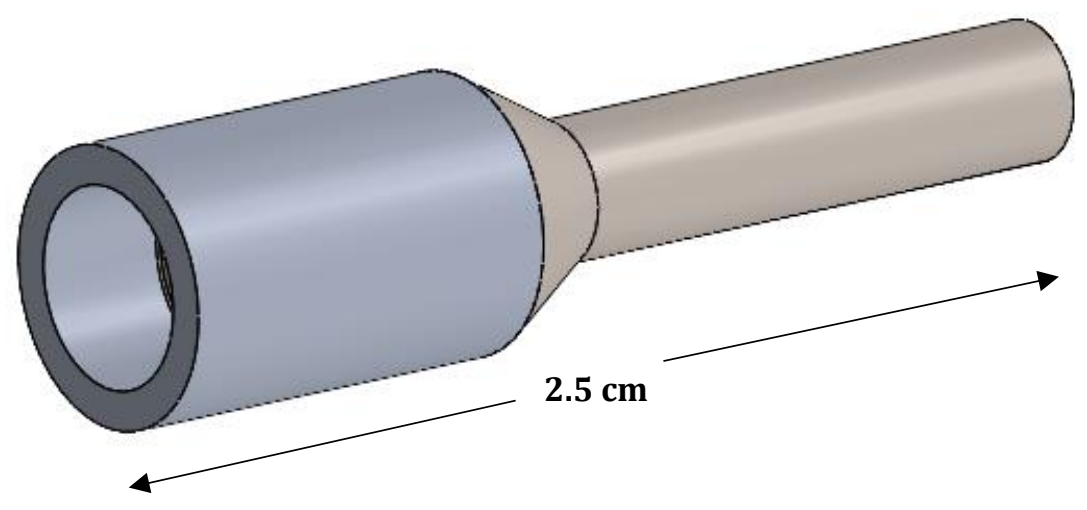

Figure 15 - Titanium to CRES reducing union, with titanium on the left and CRES on the right. 


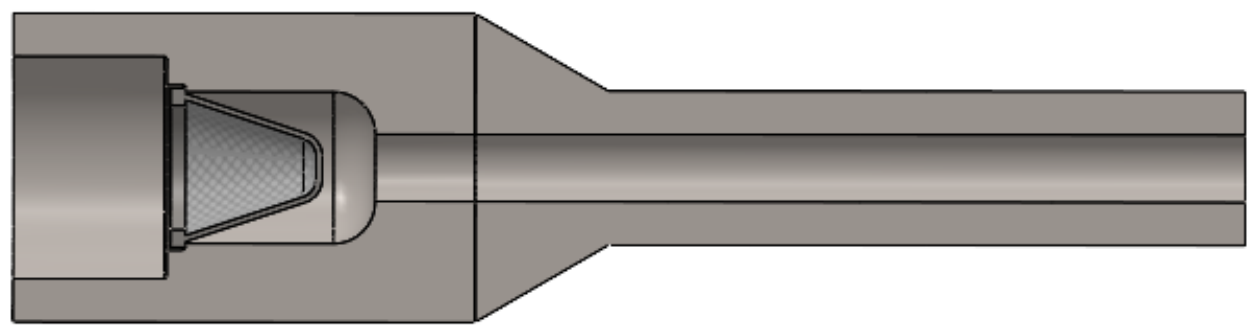

Figure 16 - Cross section of the transitional reducing union, showing the internal FOD filter.

\subsubsection{Thruster Design}

The thruster design created during Phase I (seen above in Fig. 6), was also an initial representation that required further refinement. The cross section shown in Fig. 17 has an AR provided nozzle made out of Inconel, with two AR provided bedplates to contain the catalyst. The walls are thicker than needed, creating a large thermal mass that would decrease overall system efficiency by serving as a heat sink that reduces the chamber temperature. Connecting this thruster to a thermal standoff had not yet been considered, and was an area for improvement within the current design.

The thruster design effort led to the final design shown in Fig. 18 (next page). This final design includes eight individual parts made out of Inconel that are welded together, and was designed to minimize thermal losses. The overall length of the thruster was reduced by using only one bedplate for holding the catalyst in the chamber. To prevent catalyst particles from moving upstream,

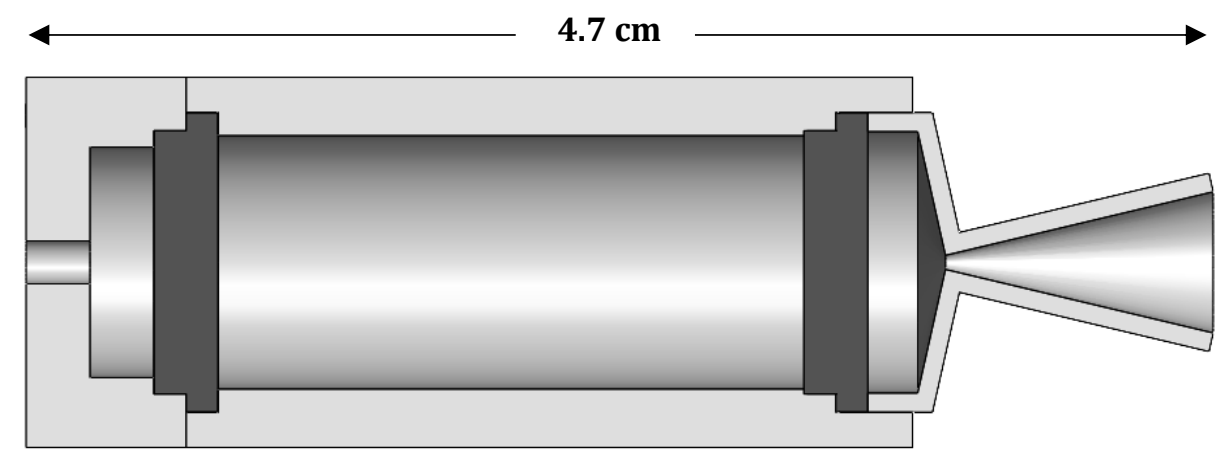

Figure 17 - Cross section of Phase I thruster design [3]. 

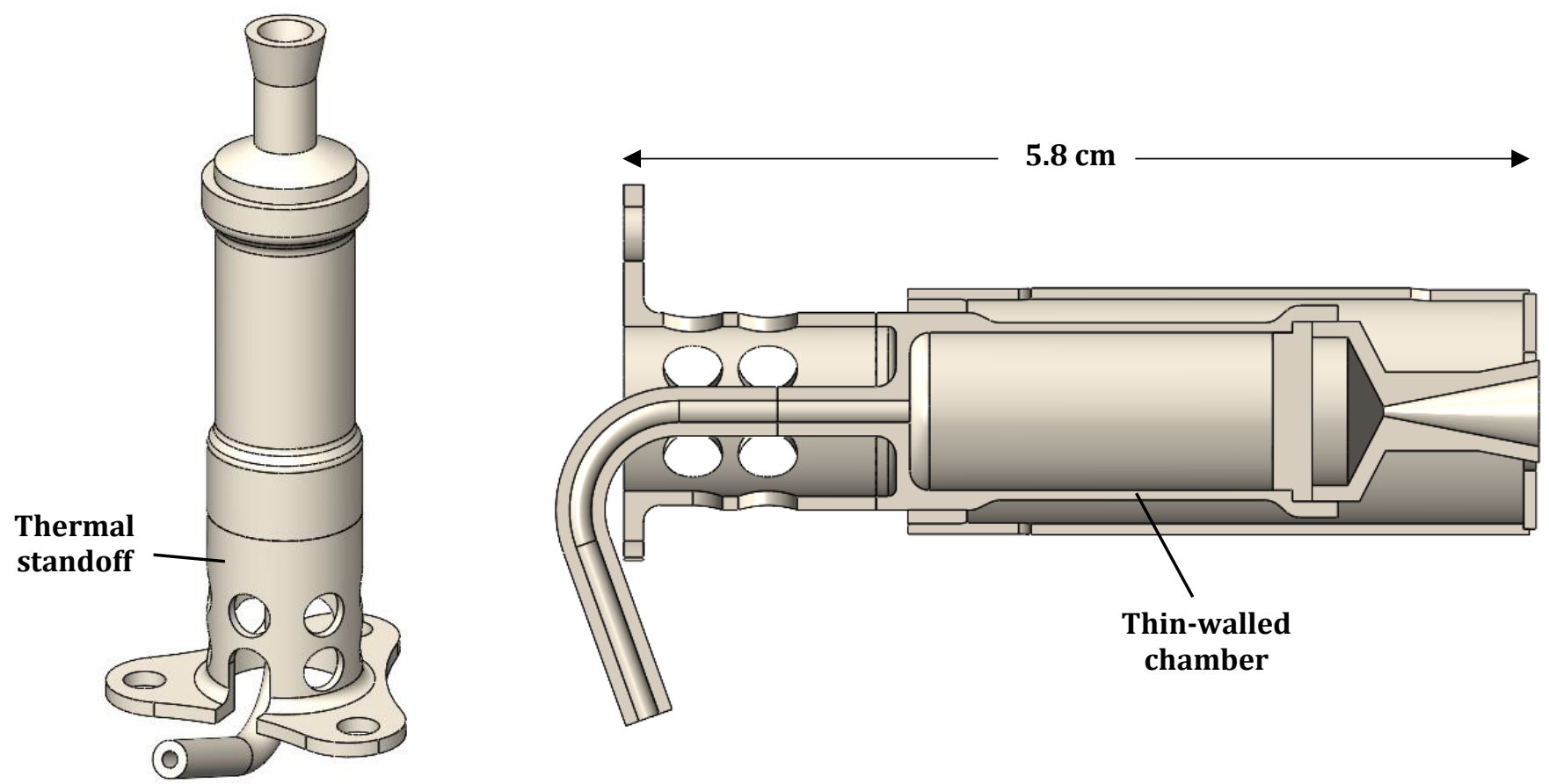

Figure 18 - Final thruster assembly without heat shield (left), and cross section of thruster assembly (right).

a circular screen is placed between the catalyst and chamber inlet during catalyst packing. The heat shield (shown attached to the thruster assembly in Fig. 19) has minimal openings and is used to reduce radiative heat loss, while the thermal standoff is used to reduce conductive heat loss. Using the hoop stress equation (Eq. 1), it was calculated that the chamber walls have a 6X factor of safety (FoS) for a chamber pressure of $P=200 \mathrm{psi}$ and wall thickness, $t=0.025$ ". In the case of failure of one of the valves, the system could still be operated (with significant losses) at a chamber pressure of 1000 psi, in which case the FoS is $1.2 \mathrm{X}$. That advantage of using thin walls is that the decreased thermal mass of the chamber allows the thruster to reach an equilibrium temperature faster during operation. Heating times and equilibrium temperatures were thoroughly tested and documented during Phase I of this effort [3].

$$
\sigma_{\theta}=\frac{P D}{2 t}
$$




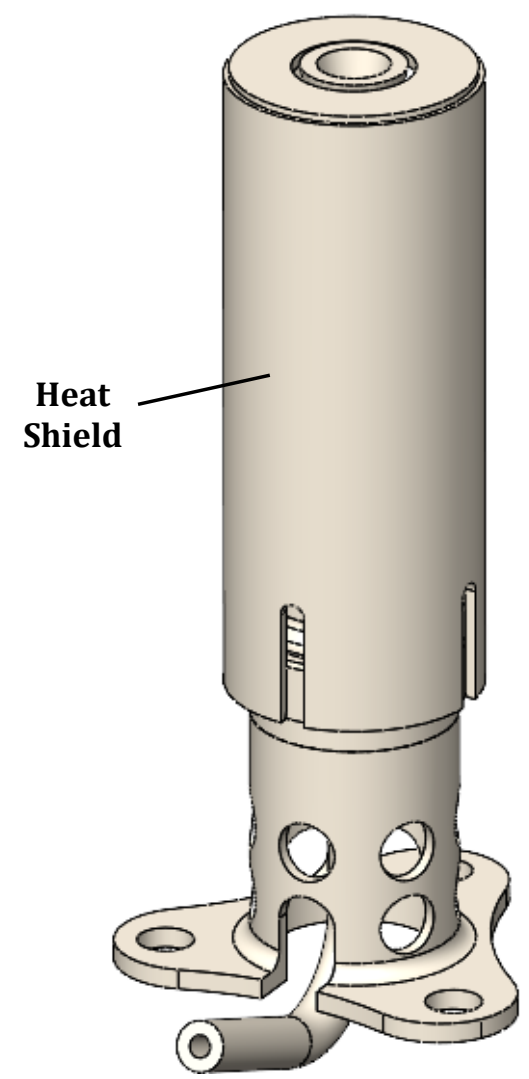

Figure 19 - Thruster assembly with attached heat shield and cap.

\subsection{Final Design of Prototype}

The final, space-compatible layout is presented in Fig. 20 through Fig. 23, and successfully addresses all of the major constraints discussed in Section 2.1. The entire design fits within a $1.5 \mathrm{U}$ footprint with room for tank elongation, the number of components has been minimized by including fully-welded connections and bolting components directly into the threaded component plate, and the operating pressure of 10,000 psi was considered throughout the design. Additionally, careful consideration was taken to ensure that there was sufficient space between components in order to perform all the necessary orbital welds. 

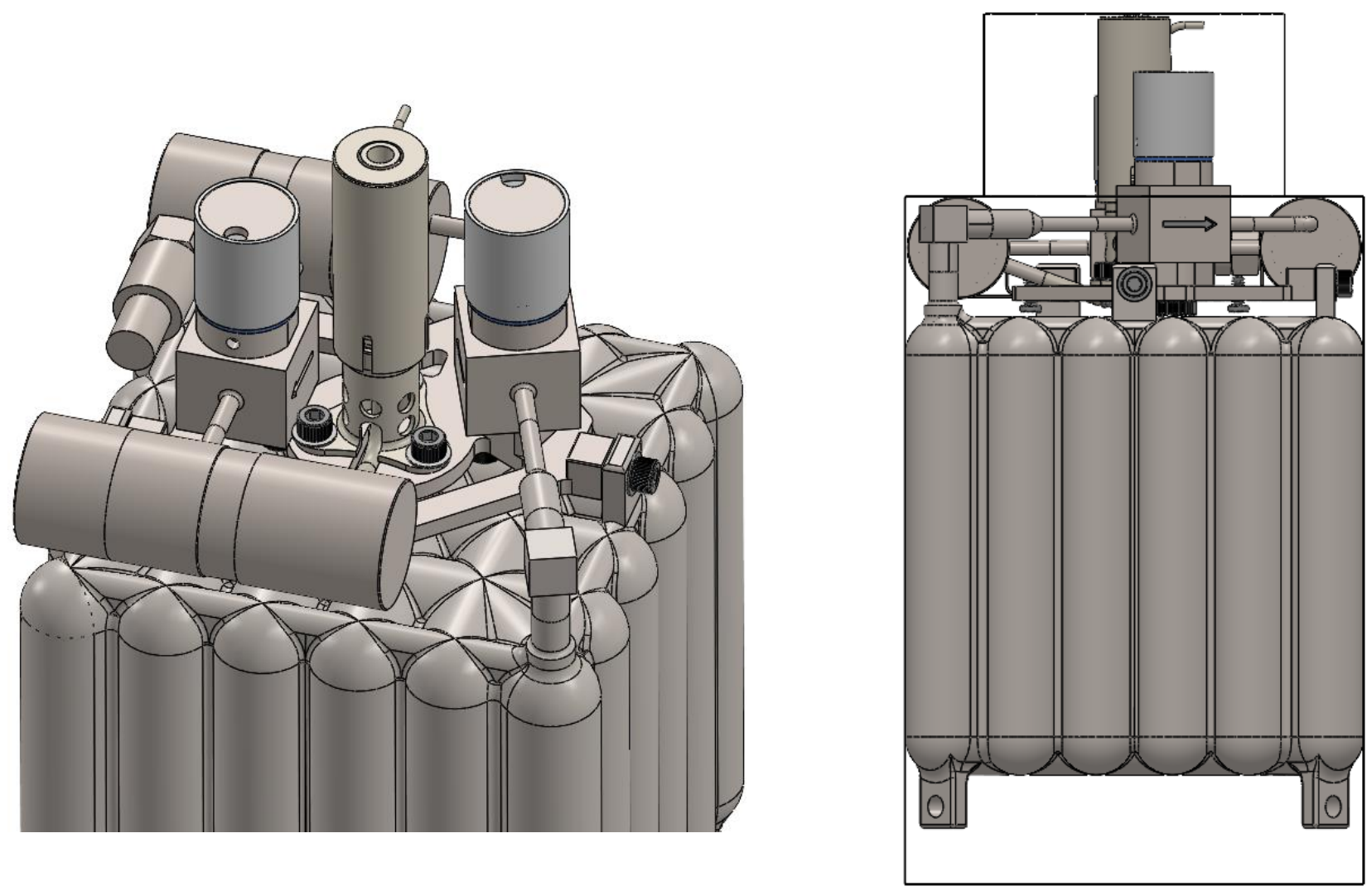

Figure 20 - Two views of the final, space-compatible layout.

The prototype, however, will be limited to an operating pressure of $1250 \mathrm{psi}$, due to the pressure rating of the off-the-shelf calibrant valves that are used in the design. Suitable, compact, offthe-shelf valves capable of 10,000 psi were not available, so this limitation leaves room for further improvement. Either valves capable of higher pressures will need to be obtained or designed, or the operating pressure of the final system will need to be decreased with considerations of the performance impact of such changes. Some options for decreasing the operating pressure of the system are discussed in Section 6. 


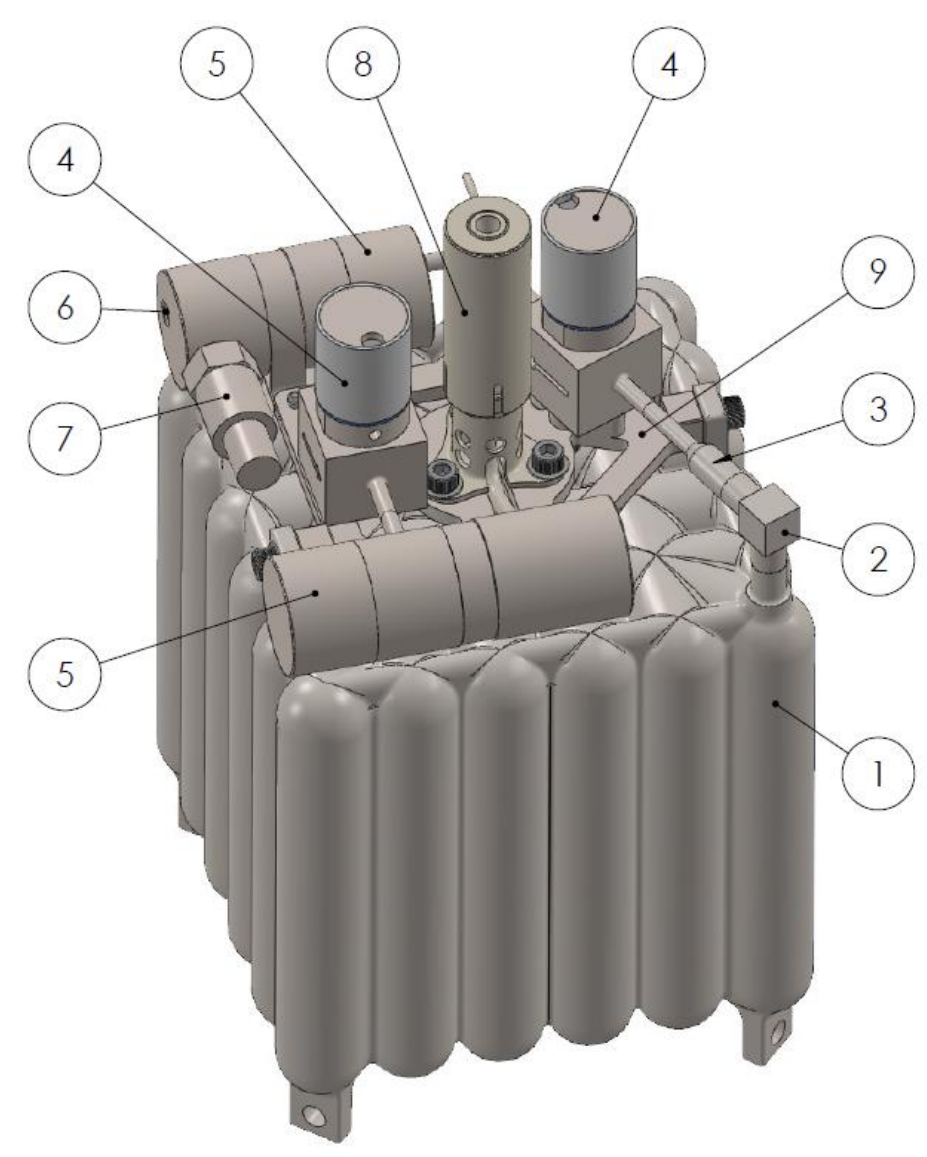

Figure 21 - Final design with labeled components.

The components of the prototype labeled in Fig. 21 are: (1) Aerojet Rocketdyne's custom engineered, additive manufactured titanium pressure vessel capable of 10,000 psi, (2) a 1/4" to 1/4" $90^{\circ}$ elbow made out of titanium (3) a custom $1 / 4^{\prime \prime}$ to $1 / 8^{\prime \prime}$, titanium to stainless steel reducing union, (4) two Parker 009-0172-900 calibrant valves capable of 1,250 psi, (5) two custom stainless steel accumulator volumes, (6) an AFO plug used to seal the system's fill/drain port, (7) a GP:50 compact flight heritage pressure transducer to monitor system pressures, (8) a custom Inconel thruster with thermal stand-off, heat shield, catalyst filled chamber, and Aerojet Rocketdyne provided nozzle, and (9) a custom stainless steel component plate to mount and attach all components to the tank. 


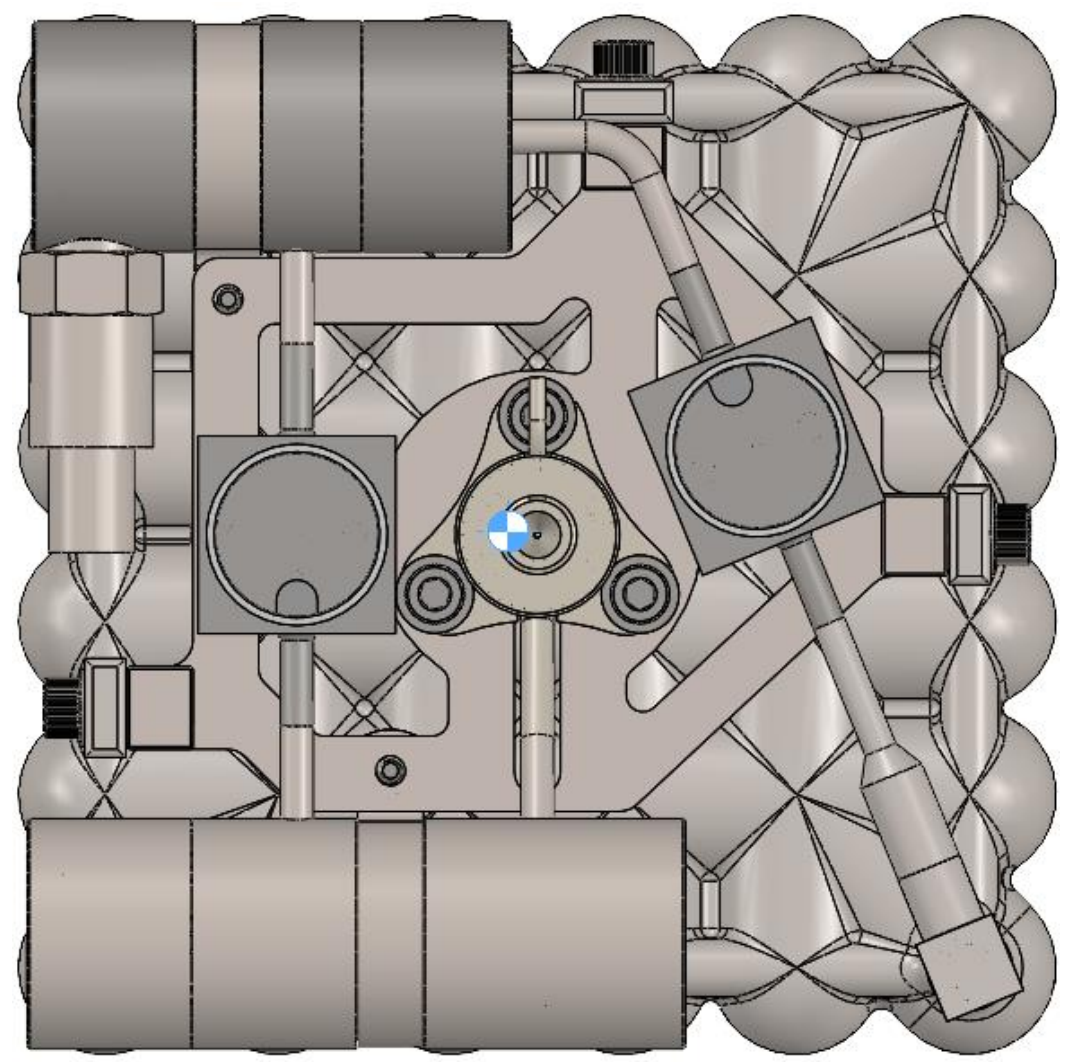

Figure 22 - Center of gravity (in blue).

The internal structure of the titanium pressure vessel is proprietary information, so the mass of the tank is estimated at $20 \%$ of the overall solid volume mass. With this estimate, the tank has a mass of $1.35 \mathrm{~kg}$ and the system has a total dry mass of $1.83 \mathrm{~kg}$ and wet mass of approximately $2.4 \mathrm{~kg}$ with 10,000 psi tridyne propellant $(88 \% \mathrm{~N} 2,8 \% \mathrm{H} 2$ and 4\% 02). The CG (shown in Fig. 22) is 0.285 $\mathrm{cm}$ left of center in the $\mathrm{x}$-direction, $0.043 \mathrm{~cm}$ above center in the $\mathrm{y}$-direction, and will vary in the $\mathrm{z}$ direction based on the length of the propellant tank, though the system can be easily ballasted to align the CG with the thruster axis.

Renderings of the final, space-compatible design were created in SolidWorks, and are presented in Fig. 23. These renders represent what the final system will look like upon completion of assembly at the end of this effort. 

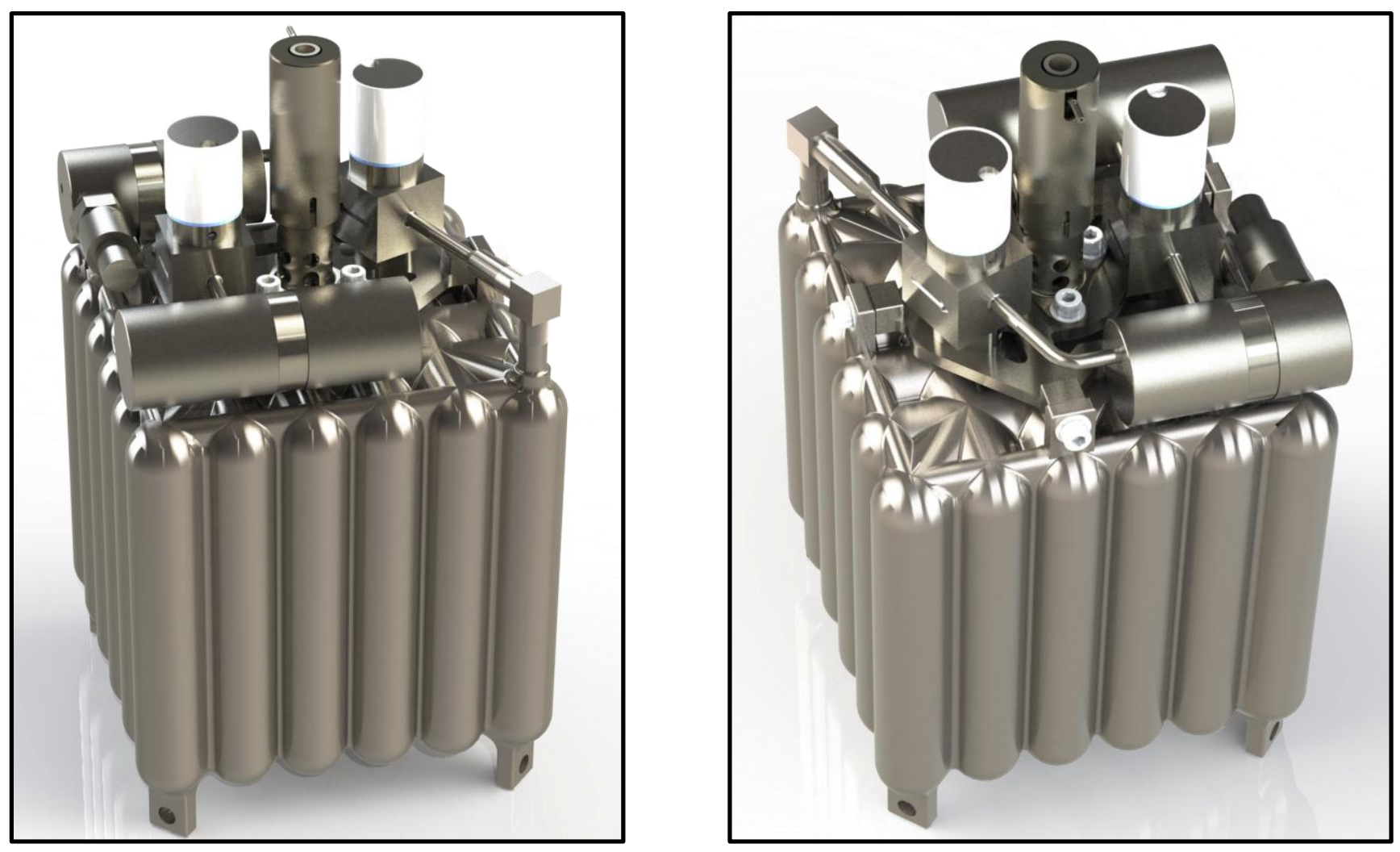

Figure 23 - 3-D renders at different angles representing the final, space-compatible system design. 


\section{Manufacturing the Prototype}

Given the many custom parts to fabricate, manufacturing the prototype was done by multiple agents. The propulsion system as presented in Section 2 has 15 parts that either require fabrication or modification, and additional tooling pieces are needed for assembly. Five of the parts were built in UW campus shops, the tank is 3-D printed by AR, and the remaining parts were built were sent to instruments shops on campus for fabrication.

\subsection{Fabricating Custom Parts}

The five parts machined by the UW team include the component mounting plate, the titanium to CRES reducing union, and a tooling plate and two-piece stand for weld assembly. The reducing union and weld assembly stand were machined on a manual lathe, while the mounting and tooling plates are more complex pieces that require computer-aided manufacturing (CAM) to fabricate. Fusion360 was used to create toolpaths for a CNC milling machine for both tooling plates. Figures 24 through 26 show a typical setup and a single adaptive clearing toolpath from Fusion360, the part being machined by the $\mathrm{CNC}$ mill, and finally, the completed tooling part.
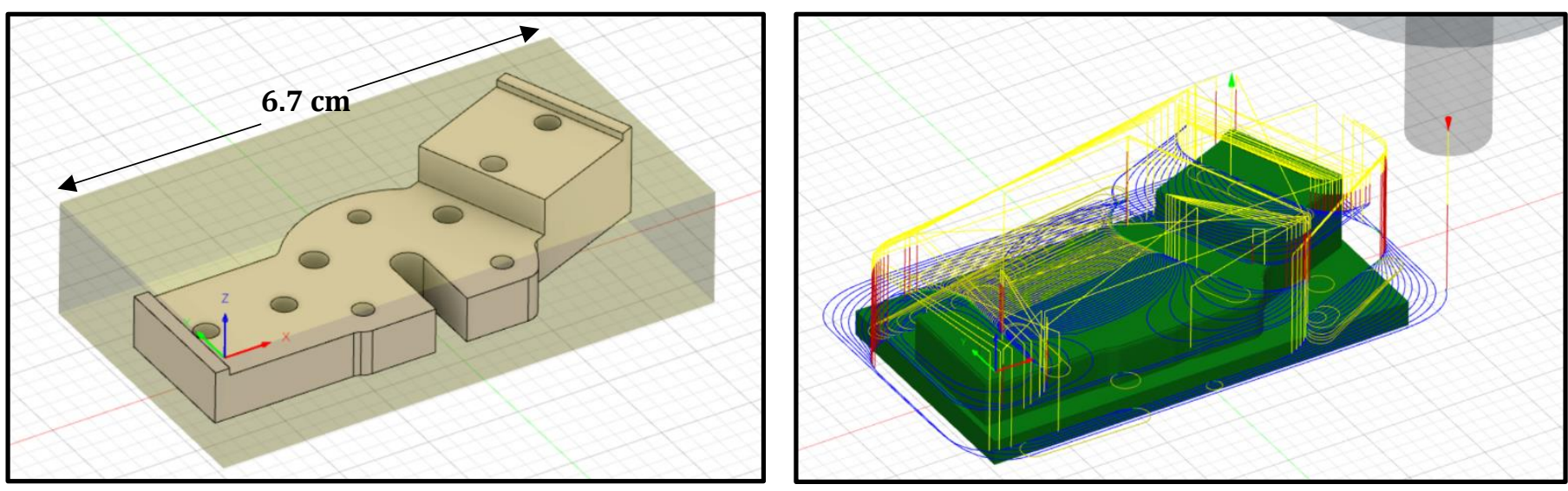

Figure 24 - Fusion360 setup showing the stock metal and tooling plate within (left), and a single step of the multistep CAM process (right). 


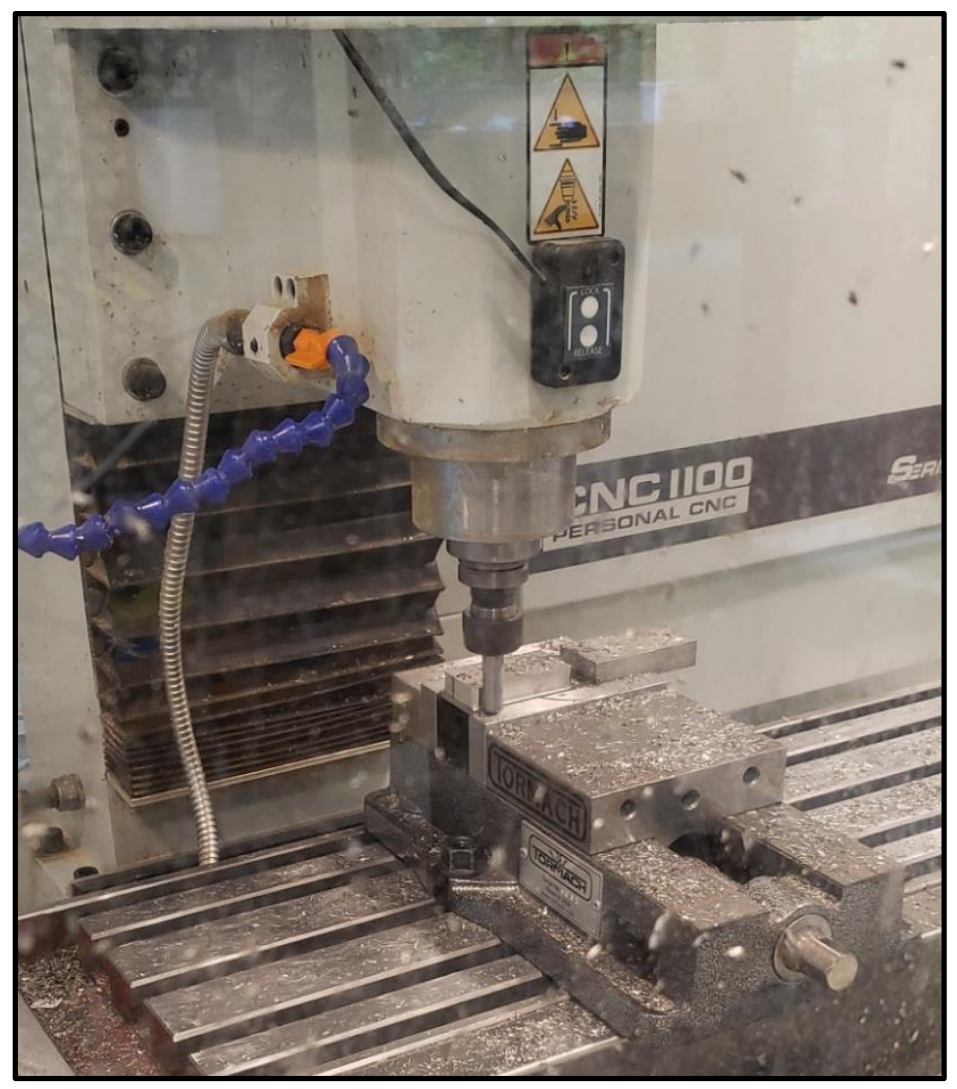

Figure 25 - Shot of the tooling plate being machined on a CNC mill.

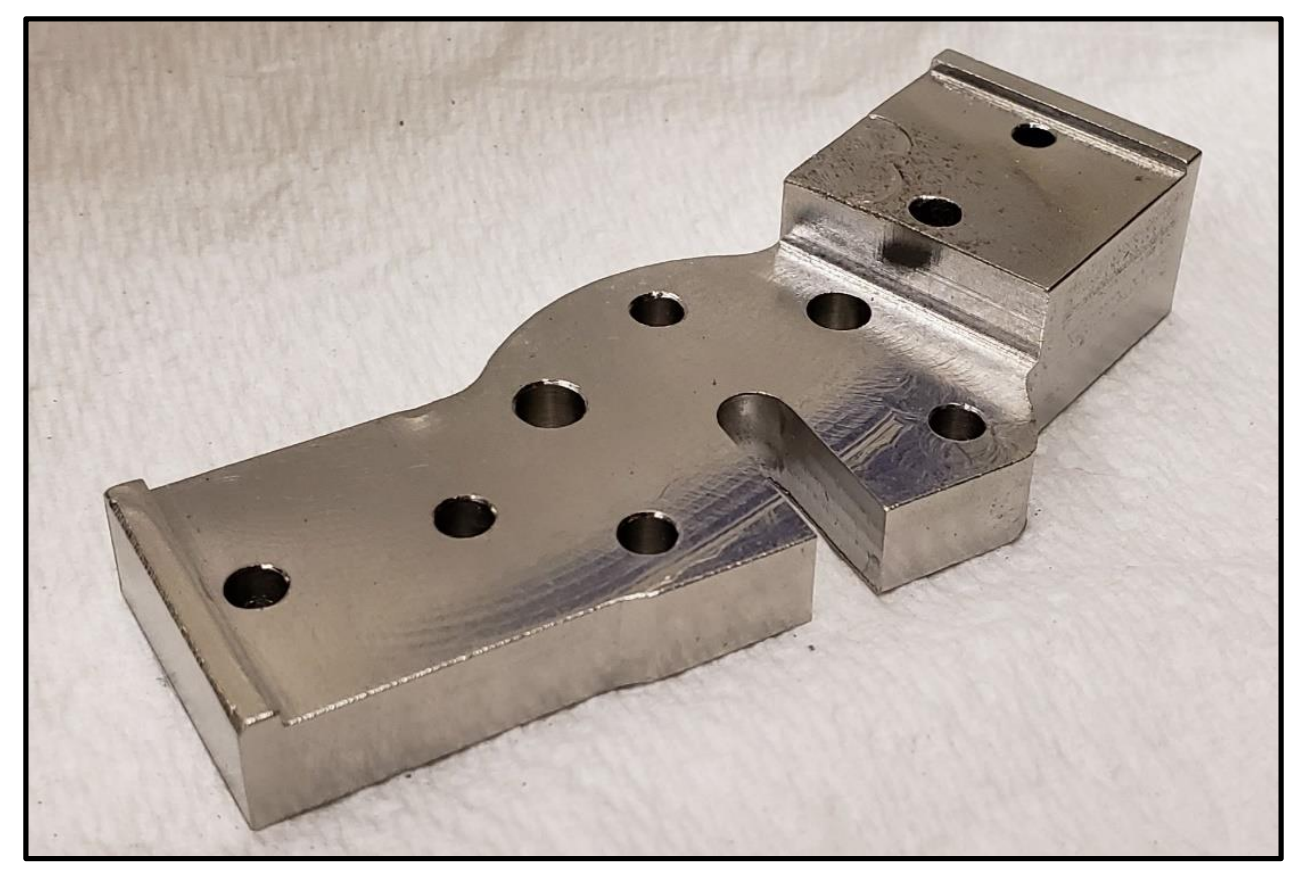

Figure 26 - Stainless steel tooling plate after completion of all CNC operations. 


\subsection{Part Drawings}

The parts that were sent out to UW campus shops for fabrication all required individual toleranced 2-D drawings, to properly convey the design intent to the machinist who would be making the piece. The drawing has to reflect that the dimensions of some features are critical and others can be less accurate. The complexity of these drawings can be seen in Fig. 27, which shows the drawing that was created for the thruster standoff. In total, between all the individual custom parts and subassemblies along with the assembly tooling pieces, over 20 toleranced drawings were created to be sent out to the shops.

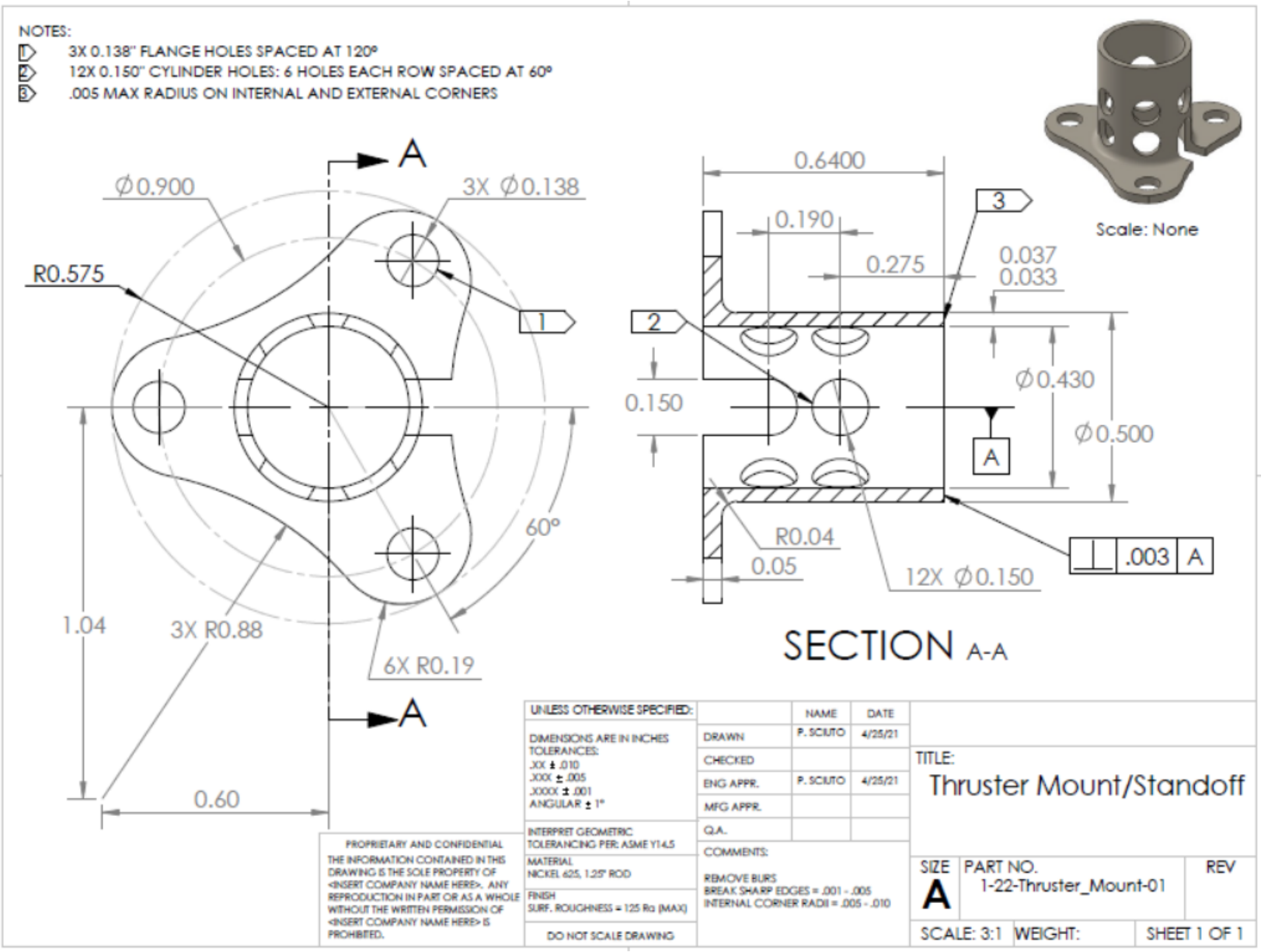

Figure 27 - 2-D drawing of the thruster standoff. 


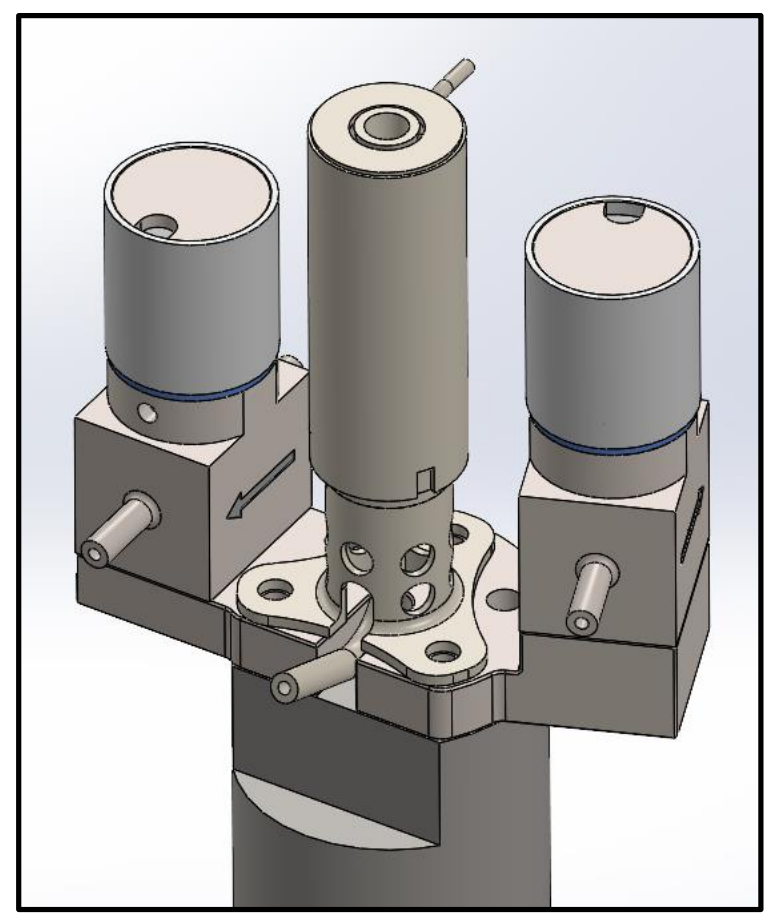

Figure 28 - Thruster assembly and valves bolted to the tooling plate/stand for welding.

\subsection{Assembly Tooling and System Assembly}

During the design process, how the prototype was going to be assembled had to be considered. Since the alignment of all the major components has to be precise, a tooling plate and stand (shown in Fig. 28) were developed and built to properly align the parts during welding. The
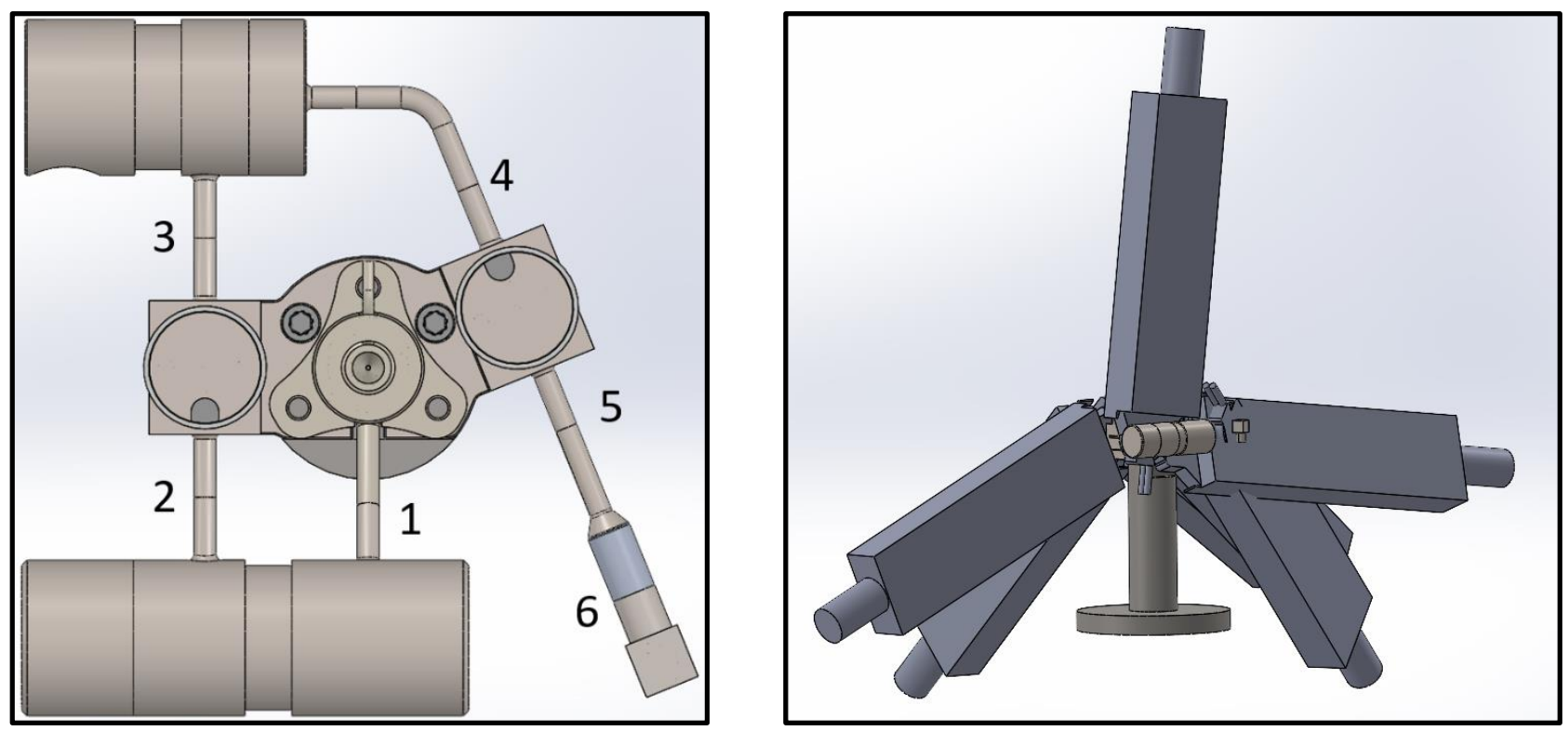

Figure 29 - The order of welding (left), and ensuring the weld head has clearance for all welds (right). The weld head has an approximate length of $30 \mathrm{~cm}$. 


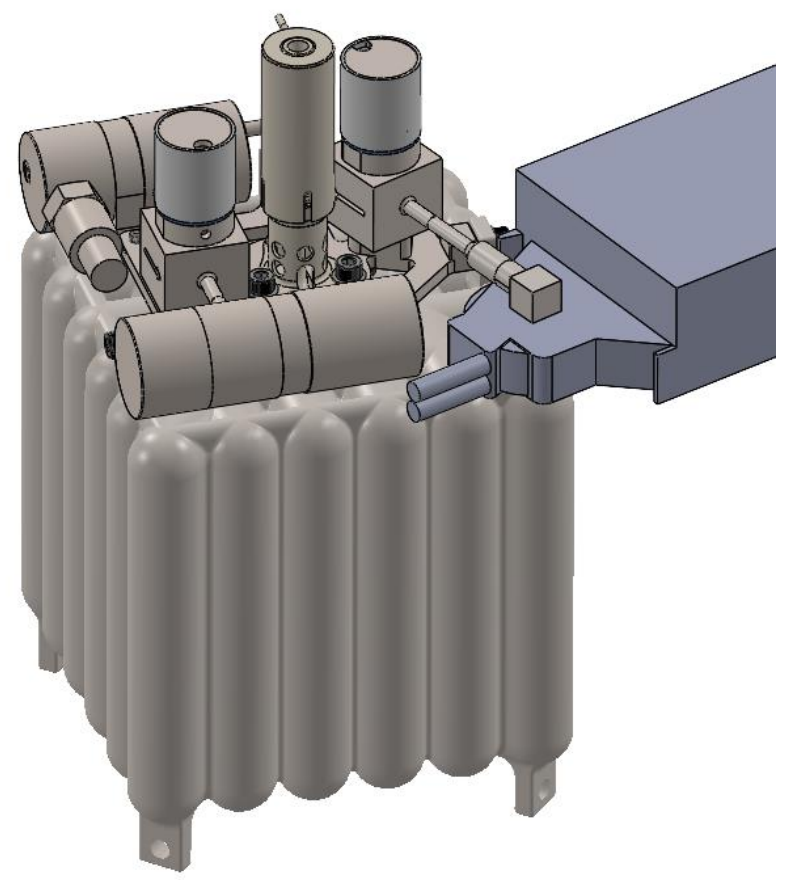

Figure 30 - After bolting the plate to the tank, the final weld is performed.

weld order was determined and a model of the weld head was placed at each weld location (Fig. 29) to ensure there was adequate clearance for the welder. Upon completion of these welds, the components are transferred to the component plate which is then bolted to the tank. Finally, the final weld between the tank and the titanium $90^{\circ}$ is performed and the propulsion system is ready to be filled (Fig. 30). 


\section{System Operation}

Bench top testing done during Phase I demonstrated the successful reaction of tridyne as it flowed through the catalyst packed chamber, without the need for preheating the chamber [3], with system pressures managed by a standard regulator connected to a gas cylinder. Using two valves in a pulse-modulated bang-bang configuration for the CubeSat propulsion system not only enables pressure control, removing the need for a pressure regulator, but also makes the system easily restartable, capable of providing small impulse bits, and allows pulsed operation to heat the thruster if needed. Simplified, one-dimensional flow calculations were done to estimate the valve timing, accumulator volumes and system pressures. Since the thruster is fixed and a gimbal is not used, realistic usage in space is discussed.

\subsection{Pulse-Modulation for Pressure Control}

Due to mass flow choking, pressure throughout the propulsion system can be controlled by rapidly pulsing the solenoid valves. Since orifice diameters within the valves are known, the maximum mass flow rate can be calculated (calculations discussed in Section 4.2). Based on plenum volumes, the fill and drain times for each volume can be estimated.

When considering the PID shown in Fig. 15, cycling the valves at a frequency of $50-150 \mathrm{~Hz}$ allows the supply pressure of 10,000 psi in the tank to be sequentially reduced to roughly $1000 \mathrm{psi}$ in the first accumulator, 400 psi in the second accumulator, and finally, 200 psi in the thrust chamber. To maintain 200 psi in the chamber, the valves have the potential to be dynamically controlled based on the current system pressure. As the supply pressure decreases to the point where the flow is no longer choked at the first valve, the valve can be cycled more slowly, still maintaining 1000 psi in the first accumulator. Once the system pressure is low enough that the flow is no longer choked through the second valve, that valve's timing can be reduced to maintain $200 \mathrm{psi}$ in the chamber. When the 
supply pressure reaches 200 psi, the system can then be operated in a blow-down mode with decreasing thrust.

The obvious advantage of pulse-modulated operation is that the chamber pressure is quasisteady, providing quasi-steady and reliable thrust. Since high-speed valves are used for the system, an added benefit is the ability to provide very small impulse bits by cycling the valves for short lengths of time, making the system ideal for maneuvers requiring high precision. Additionally, since the system operates at its highest efficiency when thermal equilibrium is reached and negligible heat is lost to the walls, thrust can be pulsed until the system is heated for higher $\Delta V$ maneuvers.

\subsection{Calculated System Pressures and Valve Timing}

To determine what volume was required for the plenums and confirm that the valves would cycle fast enough, simplified and idealized 1-D flow calculations for choked mass flow were performed using Eq. 2 [5]. In the equation, $A$ is the area at the choking point, $p$ is the pressure and $T$ is the temperature upstream of the choking point, $\gamma$ is the specific heat ratio of the tridyne gas, and $R$ is the gas constant. This calculation was performed in MATLAB for the mass flow rate at each sequential orifice of interest (code found in Appendix A), starting with a supply pressure of 10,000 psi and valve orifice diameters of $0.030 "$.

$$
\dot{m}=\frac{A p}{\sqrt{T}} \sqrt{\frac{\gamma}{R}}\left(\frac{\gamma-1}{2}\right)^{-\frac{\gamma+1}{2(\gamma-1)}}
$$

For the calculation, choked and isentropic flow is assumed, providing constant mass flow rates for a fixed pressure, and heat effects are ignored. These assumptions decrease the accuracy of the result, but still provide a good estimate of expected values. Once the mass flow rates were found, the accumulator volumes were determined by comparing the mass flow rates to the valve response 


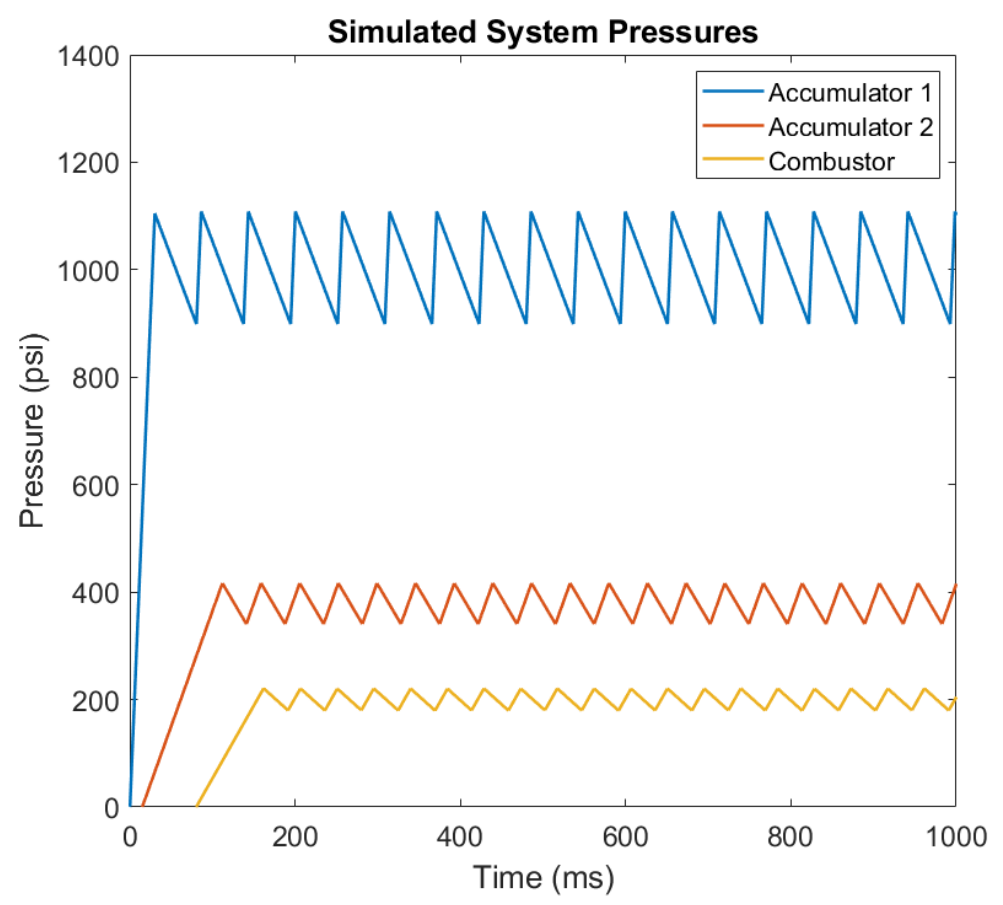

Figure 31 - Idealized system pressures throughout the propulsion system.

time of $5 \mathrm{~ms}$. It was found that the accumulators each required $0.3 \mathrm{in}^{3}$ if the valves were to cycle every $5 \mathrm{~ms}(200 \mathrm{~Hz})$. Ultimately, the chosen layout of the propulsion system allowed for larger accumulator volumes, giving the upstream accumulator a cycling frequency of $125 \mathrm{~Hz}$ and the downstream accumulator a frequency of about $35 \mathrm{~Hz}$. By decreasing the valve cycling frequency, the life of the valves is potentially extended.

With the known accumulator volumes and supply pressure known, and estimated fill/drain times for each stage, an idealized plot of the system's operation can be created (shown in Fig. 31). It can be seen that with a supply pressure of $10,000 \mathrm{psi}$, the final pressure in the chamber will range between 180 to 220 psi, providing quasi-steady thrust. These calculations are compared with bench top testing results for verification (discussed in Section 5.1). 


\subsection{In-Space Operation}

Because the CG is slightly off center (Fig. 22) and the thruster is fixed in place, how the system will operate in space must be considered. As mentioned previously, the CG could be changed with intentional placement of a small ballast mass on the opposing side, but even a slight deviation could pose a concern. Another option would be to operate the CubeSat in a spin-stable configuration, such that any off-center thrust is cancelled out by the rotation. In the case of a larger CubeSat with a multiple-U configuration, there is the potential to use multiple tridyne propulsion systems with thrusters at varying angles.

While this design effort was entirely focused on creating a CubeSat propulsion system, the low cost of the system and increased performance make a tridyne propulsion system attractive as a cold-gas replacement for other uses. Because the propellant is inert and non-toxic, the system, with some modifications, could be used in a manned maneuvering unit (MMU) for astronaut propulsion. The increased performance may also be of interest to aerospace companies currently using cold-gas thrusters for attitude control while landing spent rockets for reuse, along with any other spacecraft that may need similar attitude control. 


\section{$5 \quad$ Testing}

To confirm and characterize the effectiveness of the bang-bang pressure control, bench top testing was performed and compared with theoretical operation. The focus of this testing was on controlling pressures within the system, ultimately enabling control of the chamber pressure. With chamber pressure, chamber temperature and nozzle configuration known, performance can be estimated. Fully assembled prototype testing is planned, but will not occur until after this effort due to several manufacturing delays.

\subsection{Benchtop Testing of Pulse-Modulated Operation}

A bench top configuration using the same valves and similar system volumes as the CubeSat thruster was designed and assembled to verify system pressures in each accumulator and in the thrust chamber. Bang-bang pressure regulation testing was done with a 500 psi propellant supply, due to lab testing constraints. Further testing will be completed after this effort at a higher pressure, but still less than the designed 10,000 psi since the system pressure is currently limited by the valve's maximum pressure rating of 1250 psi. The valve timing has been characterized and this information can be used to properly control the prototype CubeSat propulsion system upon its completion.

\subsubsection{Benchtop Testing Setup}

The bench top setup was designed to directly mirror the CubeSat propulsion system's components and volumes, and is shown in Fig. 32, though tube bends are not included and a flow meter is added. The PID for the bench top test matches the CubeSat configuration's PID as shown in Fig. 3, with the addition of pressure transducers and thermocouples at both accumulators and within the thrust chamber, and the addition of the flow meter. The accumulators in this test setup are modular, enabling testing of various accumulator volumes by swapping out the $1 / 4$ " tubes. 


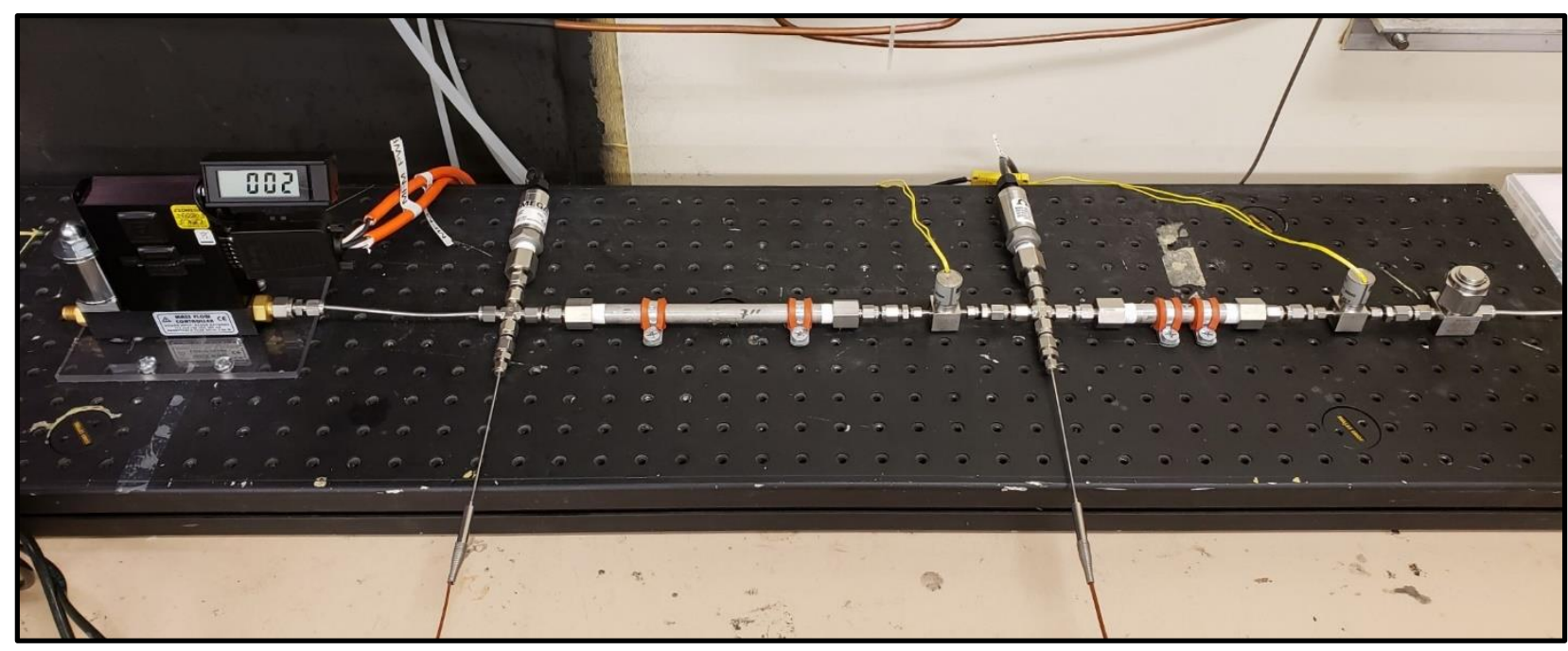

Figure 32 - Bench top setup with modular accumulator volumes on a 4' optical breadboard.

The valves are individually controlled using an Arduino Uno board wired to a MOSFET and flyback diode as shown in Fig. 33. Since the board has multiple outputs, the circuit can be duplicated for control over both valves (shown wired on a breadboard in Fig. 34). The MOSFET is set up as a switch, allowing the circuit to rapidly alternate between passing or blocking current. The flyback diode protects the components from reverse voltage spikes when the valve's coil stops being powered. The board was initially programmed in the Arduino IDE to confirm functionality and the

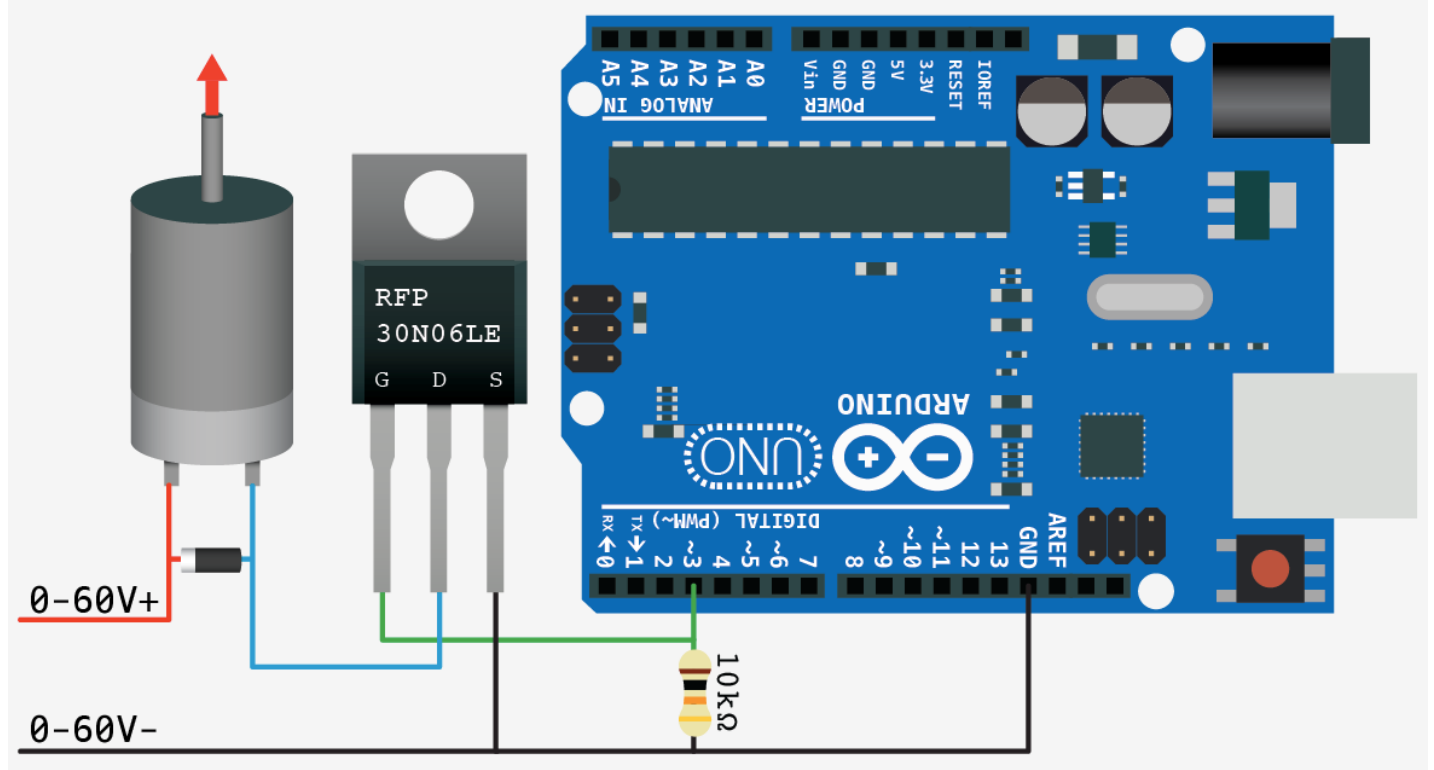

Figure 33 - Circuit diagram for single valve control with an Arduino Uno [6]. 


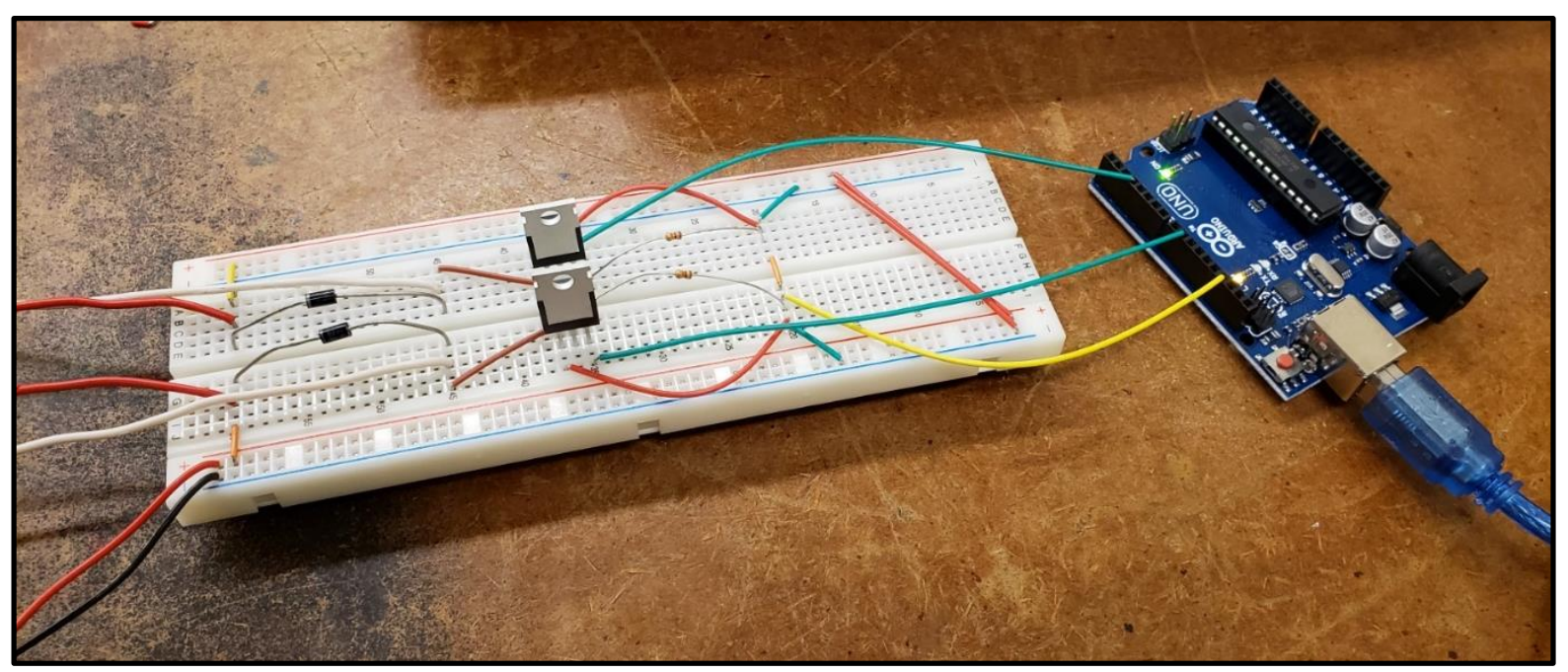

Figure 34 - Breadboard wiring connected to an Arduino Uno for dual valve control.

ability to control both valves, and after confirmation, the code was duplicated in LabVIEW to allow control and data acquisition from a common interface.

\subsubsection{Results}

Testing was performed methodically, changing only one variable per set of experiments. In the first several sets of testing, one valve was held open while the other valve was pulsed, to assess both how the system responded to a square-wave with variable frequencies and to pulse-width modulated (PWM) waves with varying duty cycles. Once individual valve performance was characterized, the valves were operated in the intended bang-bang setup, pulsing both valves at different duty cycles to demonstrate that system pressures can be controlled as intended. Note that during full pressure operation of the CubeSat at 10,000 psi, expected valve time-response will have a frequency in the range of 50 to $150 \mathrm{~Hz}$.

With the downstream valve held open and the upstream valve pulsed with a square-wave input (duty cycle, $\alpha=0.5$ ), it was found that the average pressure reduction throughout the system stays relatively constant regardless of the frequency of the square-wave, but the amplitude of the oscillations increased as the wave frequency was decreased. The pressure oscillations for a square- 


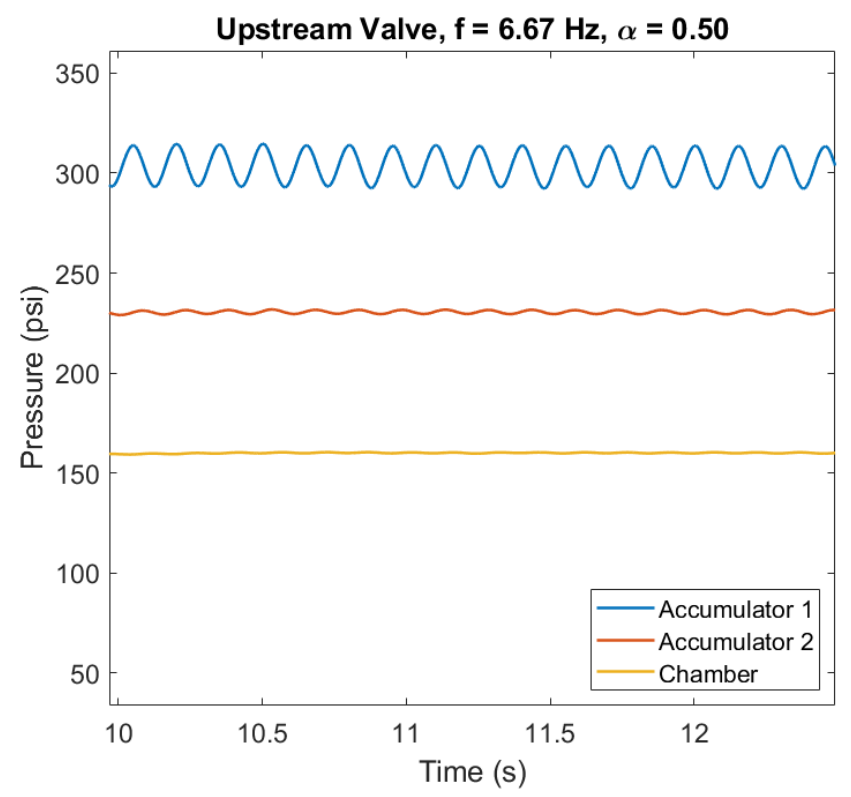

Figure 35 - Filtered pressure response with a 500 psi source, the downstream valve open, and the upstream valve pulsing with a square-wave input. Recall that accumulator 1 is the upstream plenum volume between the two valves, and accumulator 2 is the plenum volume just upstream of the thruster.

wave input with frequency, $f=6.67 \mathrm{~Hz}$ are shown in Fig. 35. This response was expected, as the valve will be open and closed for the same total time when any arbitrary square-wave is used. Testing was performed at primarily lower frequencies to better understand the response of the system. The average system pressures are shown in Fig. 36 as the square-wave frequency decreases, along with the system response with both valves held open for a steady flow. The pressures increase as the

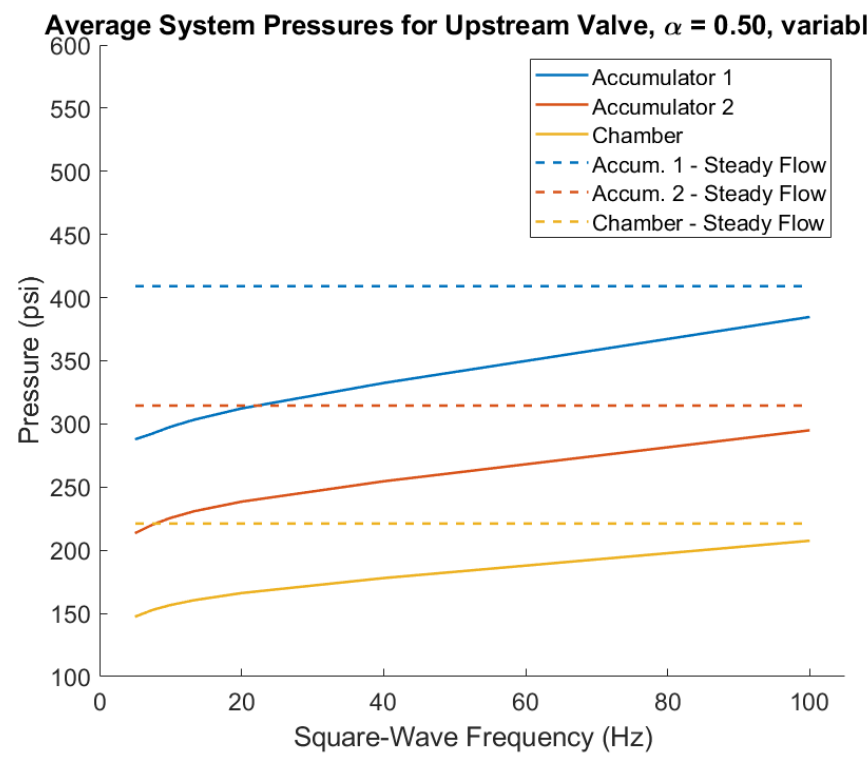

Figure 36 - Average system pressures as the square wave frequency is varied for the upstream valve, with the downstream valve held open. 


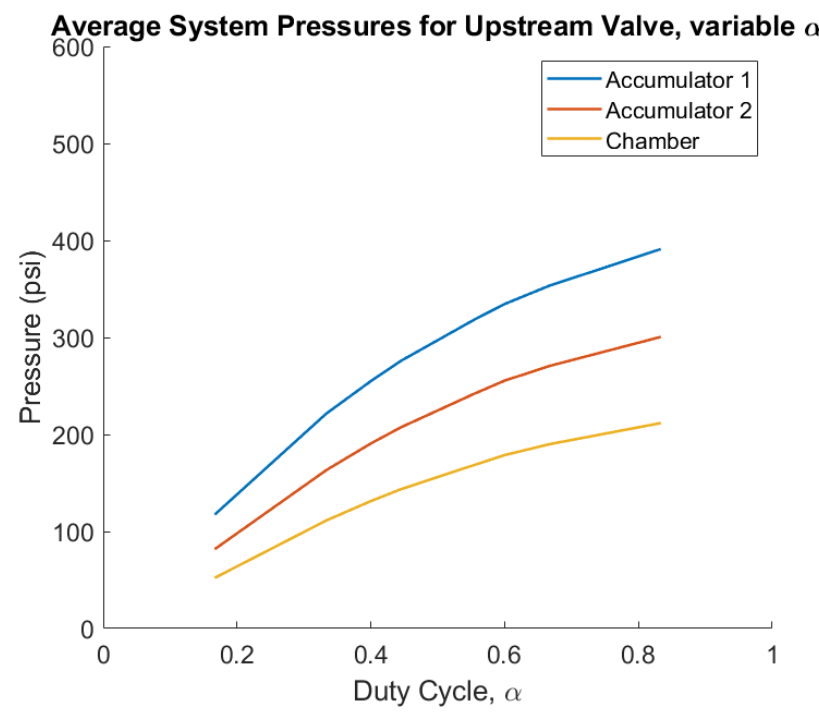

Figure 37 - Changing the duty cycle of the PWM signal allows downstream pressures to be controlled. The valve's open time was held constant at $100 \mathrm{~ms}$ for each run.

square-wave frequency increases, likely due to increased float of the valve, since the valves have a maximum rated frequency of $200 \mathrm{~Hz}$. The system response for the downstream valve was similar, showing a nearly constant pressure reduction downstream of the valve.

Similar to how an LED can be dimmed by changing the duty cycle of a PWM signal, system pressures can be controlled by changing the duty cycle of the valve. To test this, one valve was held

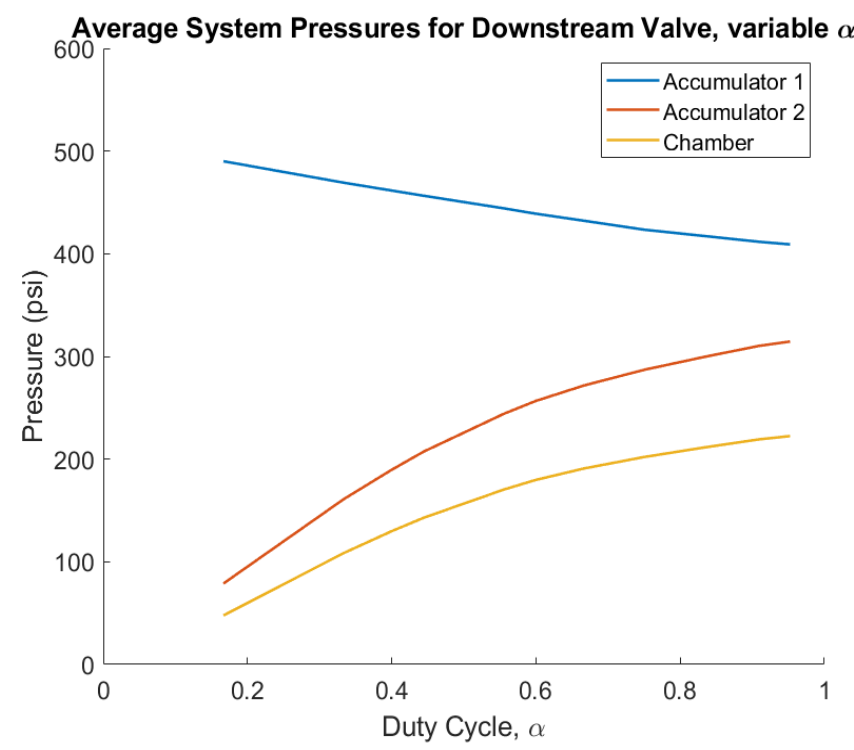

Figure 38 - System response to downstream valve duty cycle changes for PWM signal. 
open while the other valve's duty cycle was varied, first maintaining a constant 'on' time and variable 'off' time, then vice versa. For example, with the downstream valve held open, the upstream valve was opened for $100 \mathrm{~ms}$ and closed for various times ranging from $20 \mathrm{~ms}$ to $500 \mathrm{~ms}$, altering the duty cycle. As shown in Figures 37 and 38, system pressures can be controlled by changing the duty cycle of the PWM signal.

Pulsing both valves provided similar results as varying the duty cycle of an individual valve while holding the other open (discussed above and shown in Figures 37 and 38). This is expected, because the purpose of pulsing a valve is to reduce the pressure and provide a quasi-steady flow of propellant downstream of the valve. This indicates that the most effective way to reduce average system pressures is to minimize the duty cycle of each valve, effectively minimizing and maximizing mass flow in and out of each component, respectively. Since the mass flow is not truly steady (as was modeled in Section 4.2), pressure oscillations exhibit a more complex pattern. This can be seen in Fig. 39, where the downstream valve was pulsed with a square-wave input with $f=5 \mathrm{~Hz}$, and the upstream valve had a PWM input with $f=2.5 \mathrm{~Hz}$ and $\alpha=0.25$. System pressures were successfully

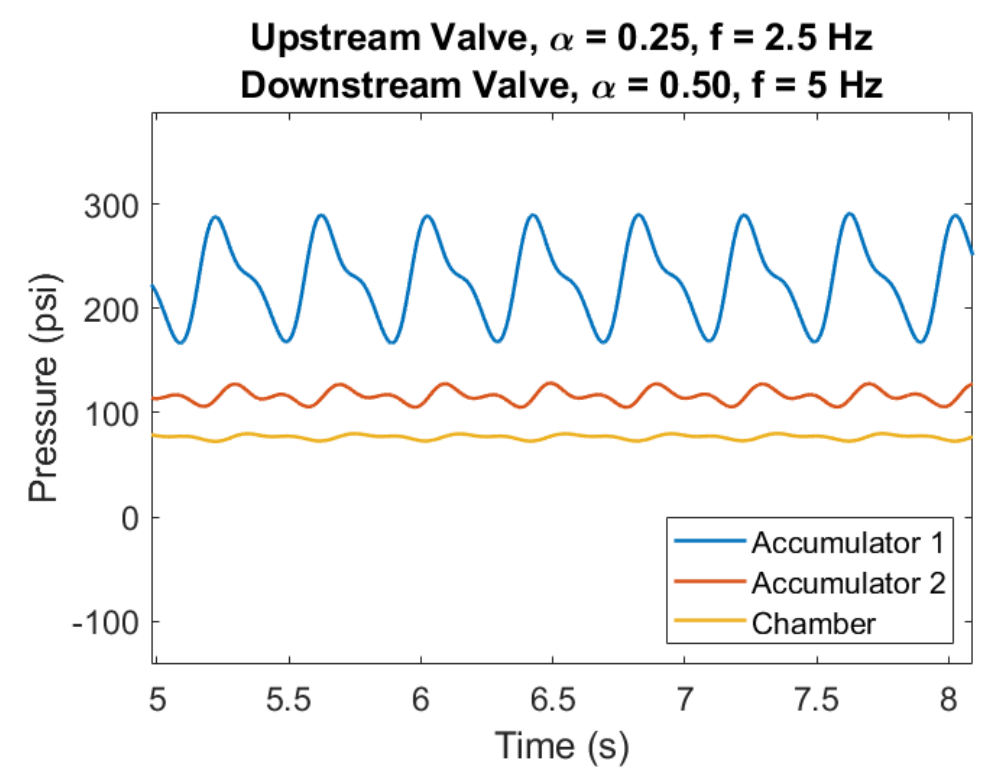

Figure 39 - Higher complexity oscillations from pulsing both valves. 
reduced from the source pressure of $500 \mathrm{psi}$, and the final chamber pressure had minimal oscillations.

From the above test results, it can be concluded that the system pressures can be effectively controlled using the bang-bang method. The duty cycle can be altered to raise or lower the downstream pressures in either accumulator, and the overall wave frequency can be increased or decreased based on the allowable pressure oscillation range in the chamber.

\subsection{Planned Prototype Testing}

Once the manufacturing and assembly of the prototype is completed, the system will be leak checked then proof pressure tested to $1.5 \mathrm{X}$ the operating pressure, or about $1875 \mathrm{psi}$, to ensure that the system can operate safely. Once proof testing is passed, the prototype will go to an unspecified Aerojet Rocketdyne customer for thorough testing and documentation, likely including system operation, vacuum, thrust, environmental, vibrational testing, and catalyst degradation assessment. 


\section{Summary}

A warm-gas thruster configuration using catalyzed tridyne propellant was developed in a configuration suitable for application on a CubeSat platform. The design includes fastresponse valves coupled with accumulators in a bang-bang configuration to provide pressure control as well as impulse modulation. The resulting design is sufficiently compact to be housed in a $1.5 \mathrm{U}$ CubeSat volume. For sufficiently high propellant storage pressure, the configuration is shown to be competitive with more complicated vaporizing-liquid thrusters, while having simplicity near that of a cold-gas system. The configuration incorporates entirely welded joints and addresses material compatibility issues. Analytical modeling indicated that valves with a 5 ms response time can stabilize the combustor chamber pressure between 180 and 220 psi for a source pressure of 10,000 psi. Preliminary experimental testing indicated that the system pressures can be reduced and effectively controlled using the bang-bang approach enabled by fast-response valves couple with accumulator volumes.

The factor currently limiting the propulsion system is the lower pressure rating of the valves. Future work could focus on increasing the working pressure of the system by modifying the current valves, or designing or purchasing custom valves. Additionally, the use of another gas as a diluent could be explored to decrease the needed storage pressure while maintaining full performance capabilities. A flight ready system may make use of both and increased working pressure as well as a different makeup of tridyne gas. 


\section{Bibliography}

[1] Gelain, Antoine. “Will SpaceX Spur Another Wave Of Smallsat Innovation?” Aviation Week Network, 10 Feb. 2021, aviationweek.com/aerospace/commercial-space/opinion-will-spacexspur-another-wave-smallsat-innovation.

[2] National Academies of Sciences, Engineering, and Medicine. 2016. Achieving Science with CubeSats: Thinking Inside the Box. Washington, DC: The National Academies Press. https://doi.org/10.17226/23503.

[3] Henderson, B., Hermanson, J.C. and Knowlen, C. "Development of a Tridyne Propulsion System for CubeSat Applications," 2021 AIAA SciTech Forum. doi: 10.2514/6.2021-2028.

[4] “ISIPOD CubeSat Deployer.” ISISPACE, 29 Dec. 2020, www.isispace.nl/product/isipod-cubesatdeployer/.

[5] “Mass Flow Choking.” NASA, NASA, www.grc.nasa.gov/www/k-12/airplane/mflchk.html.

[6] “Bildr High-Power Control: Arduino + N-Channel MOSFET.” Bildr, 5 Mar. 2012, bildr.org/2012/03/rfp30n06le-arduino/. 


\section{Appendix A}

\section{MATLAB Flow Calculations}

\section{Contents}

- 1 - Mass flow at: (1) tank exit, (2) valve 1 exit, and (3) valve 2 exit

- 2 - Filling inter-volume

- 3 -Thruster-supply volume (fill/drain cycle)

- 4-Combustor Pressure

- Display

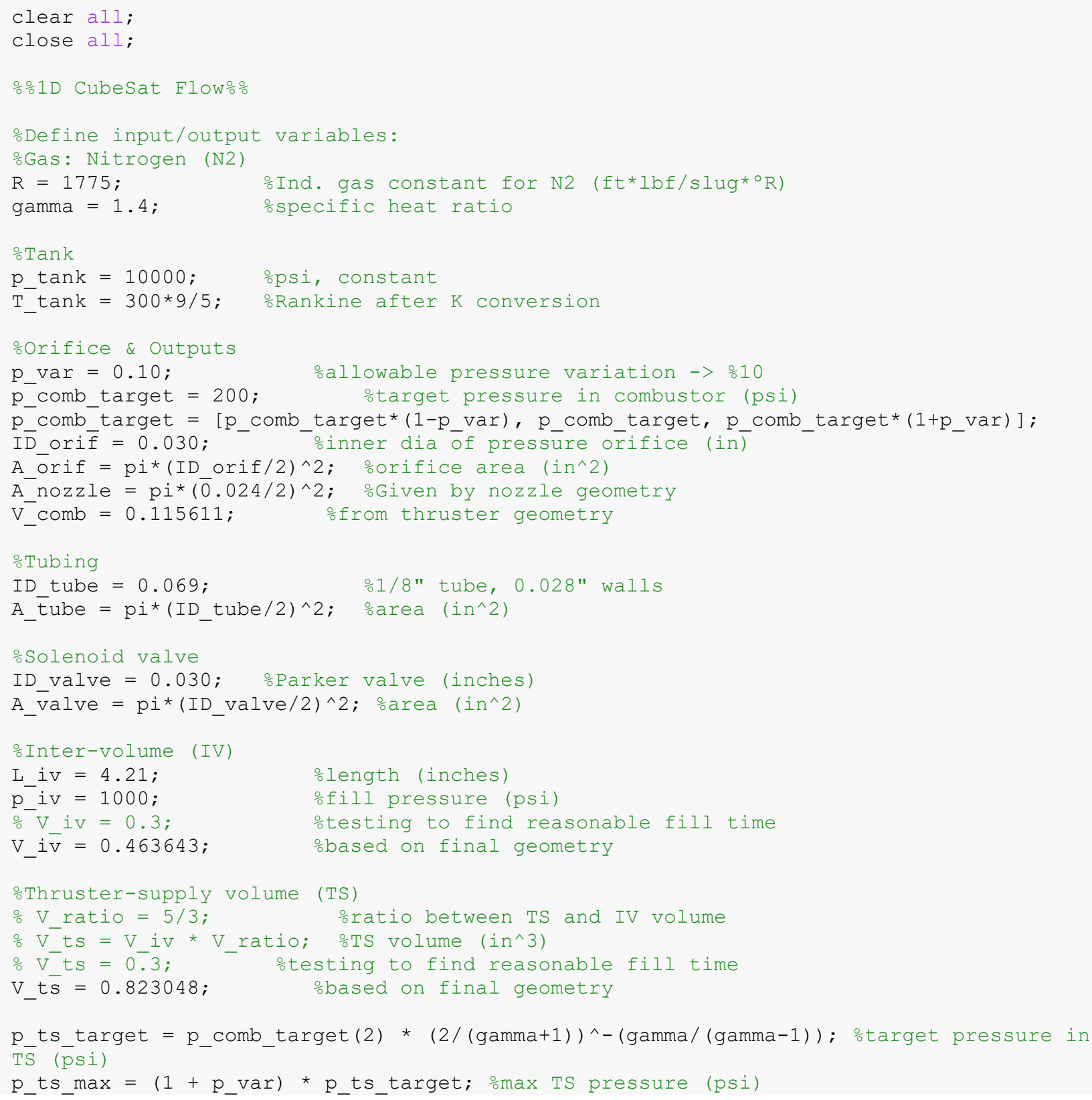


p_ts_min $=\left(1-p_{-}\right.$var $) * p_{-}$ts_target; min TS pressure (psi)

\% p_iv_target $=p_{-}$ts_target * $(2 /(\text { gamma }+1))^{\wedge}-($ gamma $/($ gamma-1) $)$; otarget pressure in IV (psi)

p_iv_target $=$ p_iv;

p_iv_max $=\left(1+p_{-}\right.$var $) * p_{-}$iv_target; omax IV pressure (psi)

p_iv_min $=\left(1-p_{-}\right.$var $) * p_{-}$iv_target; omin IV pressure (psi)

\section{1 - Mass flow at: (1) tank exit, (2) valve 1 exit, and (3) valve 2 exit}

oMass flow rate (lb/s), using equation for choked compressible flow

ohttps://www.grc.nasa.gov/www/k-12/VirtualAero/BottleRocket/airplane/mflchk.html

$\mathrm{M}=1 ;$; choked

mdot_tank $=$ A_tube*p_tank $/(T \text { tank })^{\wedge}(1 / 2) \star(\text { gamma } / R)^{\wedge}(1 / 2) \star \mathrm{M} \ldots$

$\star\left(1+0.5 *(\text { gamma }-1) * M^{\wedge} \overline{2}\right)^{\wedge}-(($ gamma +1$) /(2 *($ gamma -1$))) * 32.174$;

mdot_valve1 = A_valve*p_tank/ $\left(T_{-} \operatorname{tank}\right)^{\wedge}(1 / 2) \star(\operatorname{gamma} / \mathrm{R}) \wedge(1 / 2) * \mathrm{M} \ldots$

$\star\left(1+0.5^{\star}(\text { gamma }-\overline{1}) * M^{\wedge} 2\right)^{\wedge}-(($ gamma +1$) /(2 *($ gamma -1$))) * 32.174 ;$

mdot_valve2 $=A \_v a l v{ }^{*} p_{-} i v /\left(T_{-} \text {tank }\right)^{\wedge}(1 / 2) \star(g a m m a / R)^{\wedge}(1 / 2) \star M \ldots$

$\star\left(1+0.5^{\bar{*}}\left(\text { gamma-1) } \mathrm{M}^{\wedge} 2\right)^{\wedge}-\left((\right.\right.$ gamma +1$) /\left(2^{\star}(\right.$ gamma -1$\left.\left.)\right)\right) \star 32.174$;

mdot_orif $=$ A_orif*p_ts_target $/(T \text { tank })^{\wedge}(1 / 2) \star($ gamma/R)^(1/2) * M $\ldots$

$\star\left(1+0.5 *(\right.$ gamma $\left.-\overline{1}) * M^{\wedge} 2\right) \wedge-(($ gamma +1$) /(2 *($ gamma -1$))) * 32.174$;

mdot nozzle $=A$ nozzle*p comb target $(2) /(T \text { tank })^{\wedge}(1 / 2) \star(\text { gamma } / \mathrm{R})^{\wedge}(1 / 2) \star M \ldots$

$\star\left(1+0.5^{\bar{*}}(\text { gamma }-1)^{\star} \mathrm{M}^{\wedge} 2\right)^{\wedge}-(($ gamma +1$) \bar{T}(2 *($ gamma -1$))) * 32.174 ;$

oNote that mass flow is modeled as constant with above orifice calc,

oven though p_ts is changing

\section{2 - Filling inter-volume}

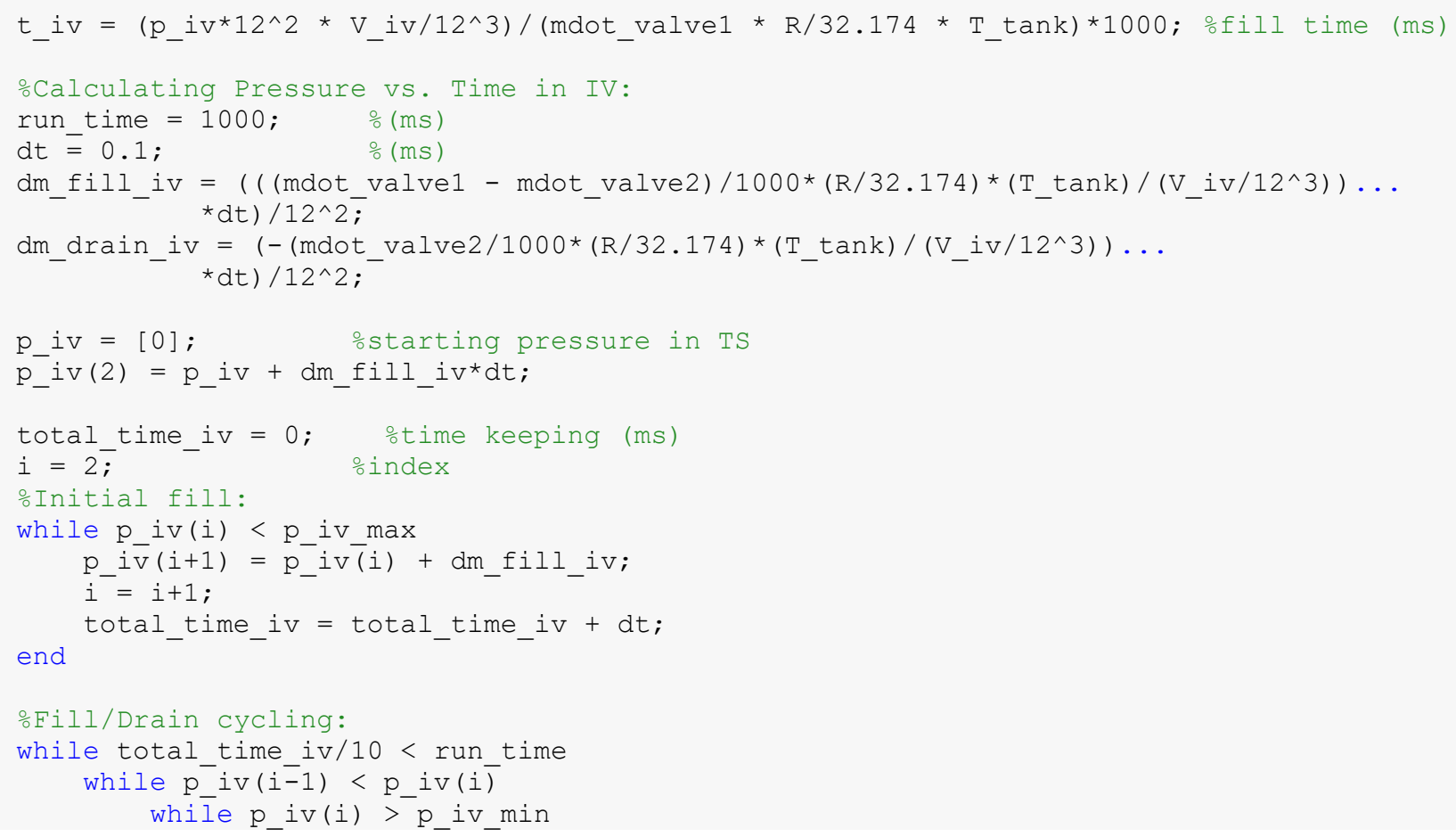




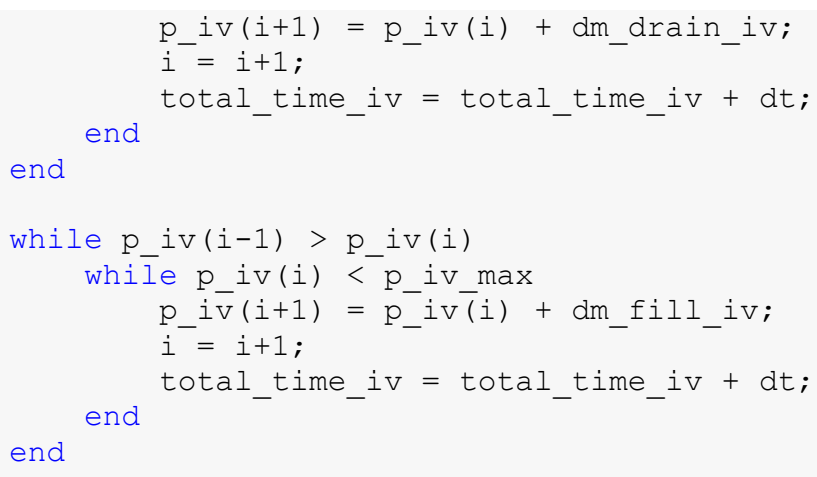

\section{3 - Thruster-supply volume (fill/drain cycle)}

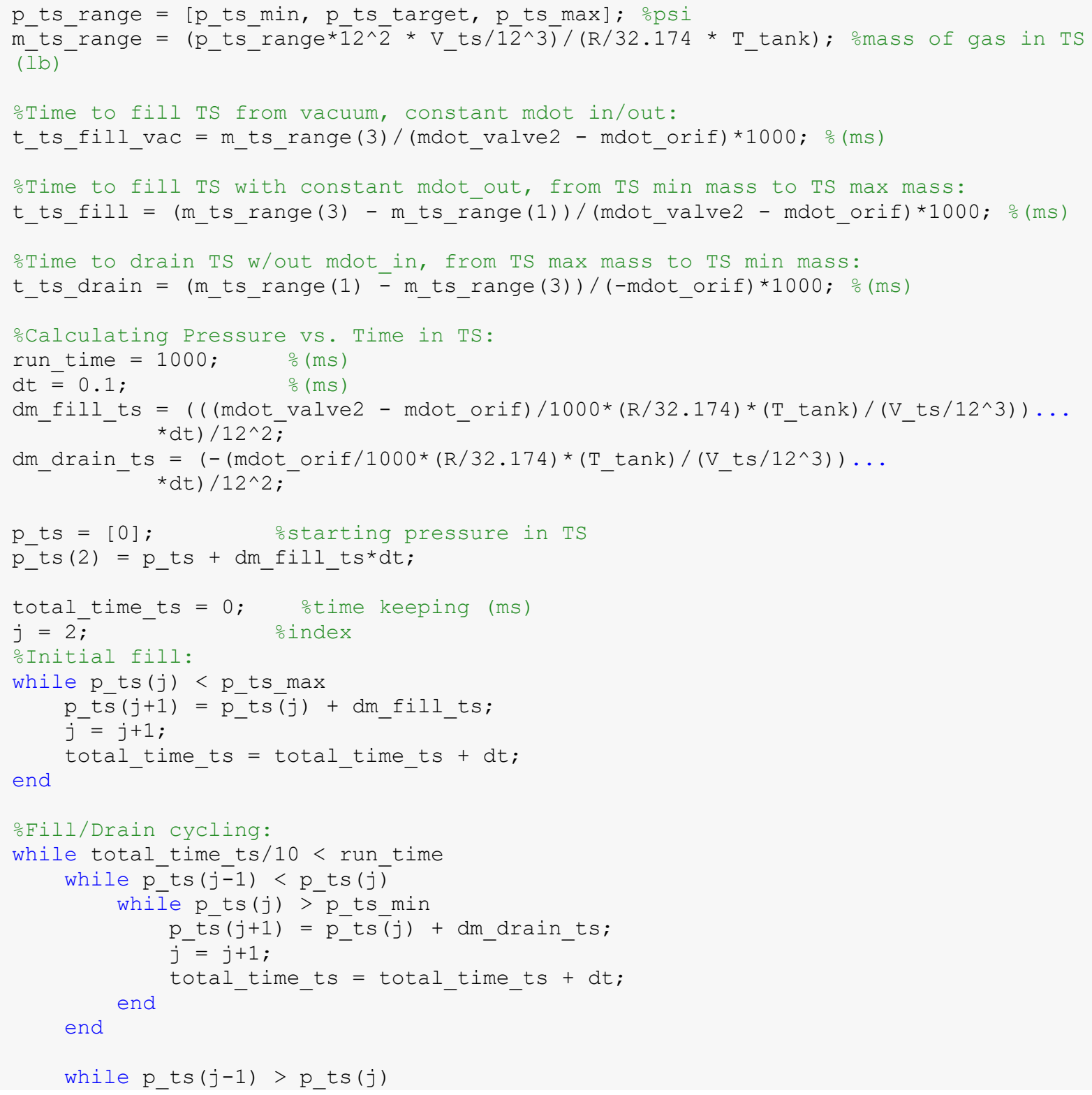




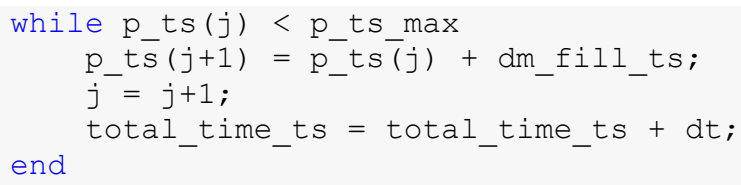

\section{4 - Combustor Pressure}

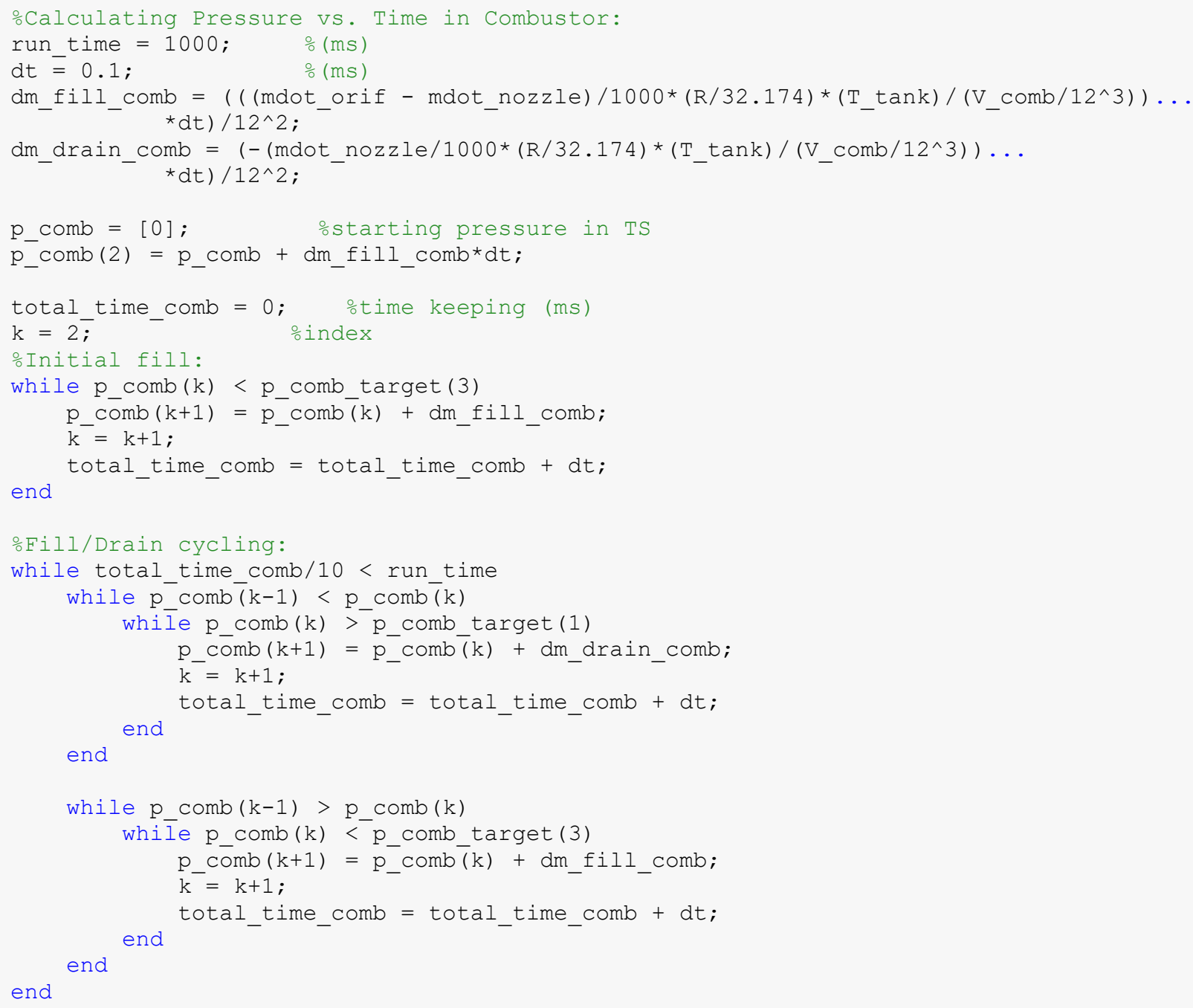

